\title{
Distributed Averaging With Random Network Graphs and Noises
}

\author{
Tao Li, Senior Member, IEEE, and Jiexiang Wang
}

\begin{abstract}
We consider discrete-time distributed averaging algorithms over multi-agent networks with measurement noises and time-varying random graph flows. Each agent updates its state by relative states between neighbours with both additive and multiplicative measurement noises. The network structure is modeled by time-varying random digraphs, which may be spatially and temporally dependent. By developing difference inequalities of proper stochastic Lyapunov function, the algebraic graph theory and martingale convergence theory, we obtain sufficient conditions for stochastic approximation type algorithms to achieve mean square and almost sure average consensus. We prove that all states of agents converge to a common variable in mean square and almost surely if the graph flow is conditionally balanced and uniformly conditionally jointly connected. The mathematical expectation of the common variable is right the average of initial values, and the upper bound of the mean square steady-state error is given quantitatively related to the weights, the algorithm gain and the energy level of the noises.
\end{abstract}

\section{Index Terms}

Distributed averaging, Multi-agent system, Additive and multiplicative noise, Random graph

\section{INTRODUCTION}

In real networked systems, there exist various kinds of uncertain factors, such as channel noises, channel fading, random link failures and recreations. In recent years, stochastic multiagent networks have attracted great attention from scholars in various fields and become an active interdisciplinary research subject. For stochastic multi-agent networks, the distributed averaging is one of the most fundamental problems and has wide application background,

This work was supported by the National Science Found for Outstanding Young Scholars, National Natural Science Foundation of China under Grant 61522310 and the Shanghai Rising-Star Program under grant 15QA1402000. Please address all the correspondences to Tao Li: Phone: +86-21-54342646-318, Fax: +86-21-54342609, Email: tli@math.ecnu.edu.cn.

Tao Li is with Department of Mathematics, East China Normal University, Shanghai 200241, China.

Jiexiang Wang is with School of Mechatronic Engineering and Automation, Shanghai University, Shanghai 200072, China. 
such as distributed computation ([1]-[2]), distributed filtering ([3]-[4]), information fusion over wireless sensor networks ([5]), distributed learning and optimization ([6]-[7]) and load balancing ([8]) etc.

The measurement/communication noises affect not only the decision-making of each individual agent, but also the overall performance of the whole system. Generally, measurement/communication noises are divided into two categories: additive and multiplicative noises. The additive noise corrupts the signal in the form of superposition regardless of the signal's own intensity. Differently, the multiplicative noise is coupled with the signal together. For example, the effects of coherent fading can be modeled by multiplicative noises in imaging radar systems ([10]). For distributed averaging with additive measurement noises, Huang and Manton ([11]) proposed the discrete-time stochastic approximation type average-consensus protocol, and gave sufficient conditions for mean square consensus under fixed undirected graphs. Li and Zhang ([12]) studied the continuous-time distributed averaging algorithm with additive measurement noises and obtained necessary and sufficient conditions for mean square average-consensus under fixed balanced digraphs. For distributed averaging with multiplicative measurement noises, Li et al. ([13]) considered the average consensus under fixed undirected graphs with nonlinear noise intensity functions, and gave the necessary and sufficient conditions for mean square average consensus. $\mathrm{Ni}$ and $\mathrm{Li}$ ([14]) considered distributed consensus with multiplicative measurement noises where the noise intensities are the absolute values of relative states.

Besides measurement/communication noises, the network structure of a multi-agent network often randomly changes due to packet dropouts, link/node failures and recreations, which are particularly serious for wireless networks. The random switching of the network structure has a strong impact on the convergence and performance of distributed averaging algorithms. This topic also attracts extensive attentions from the community of distributed averaging. Distributed averaging and consensus with independent identically distributed (i.i.d.) graph flows were considered in [15]-[20]. Especially, Bajović et al. [19] proved that the exact convergence rate in probability is exponentially fast for products of i.i.d. symmetric stochastic matrices. The cases with ergodic stationary and finite state homogeneous Markov chain type graph flows were considered in ([21]) and ([22]), respectively, which both obtained necessary and sufficient conditions for almost sure consensus. Liu et al. [23] and Touri and Nedic [24] studied distributed consensus with more general random graph flows. Liu et al. [23] obtained sufficient conditions for $L_{p}$ consensus assuming that the $\delta$-graph contains a spanning tree. Touri and Nedic [24] gave a more general condition for the convergence of weak periodic 
random matrix sequences.

Most of the above literature considered the effect of random changing of network structures or measurement noises on distributed algorithms separately. In real networks, various kinds of uncertainties may co-exist, for example, there may be additive measurement noises and channel fading accompanied with random link changes. Many scholars have long been committed to developing distributed averaging algorithms with comprehensive uncertainties, establishing convergence conditions and quantitative relations between algorithm performances and network parameters. However, as far as we know, few results were obtained on distributed averaging with the above three kinds of random uncertainties integrated together. $\mathrm{Li}$ and Zhang [25] considered the distributed averaging with additive measurement/communication noises and deterministic switching graph flows. They established the necessary and sufficient condition for mean square average consensus under fixed digraphs and the jointly-containingspanning-tree condition for mean square and almost sure average consensus under switching digraphs. Rajagopal and Wainwright [26] studied the distributed averaging with additive storage noises, additive communication noises and data-constrained communication. Kar and Moura [27] gave sufficient conditions for almost sure consensus under Markov chain type graph flows with the fixed mean graph and additive measurement noises. Huang et al. [28] considered the case with spatial-temporal-independent additive measurement noises and random link gains under Markov and deterministic switching network graph flows. They obtained sufficient conditions for mean square and almost sure consensus. Aysal and Barner [29] proposed a model of general consensus dynamics and gave conditions for almost sure convergence under additive disturbances and randomly switching graphs. Patterson et al. [30] considered distributed averaging with spatial-temporal-independent random link failures and random input noises. They gave the exponential mean square convergence rate for mean square average-consensus assuming that the underlying mean graph is always undirected and connected. Wang and Elia [31] focused on the system fragilities caused by communication constraints (additive input noises, communication delay and fading channels). They established the tight relation among uncertainties of network channels, robust mean square stability and the appearance of Lev́y flight, and gave conditions for mean square weak consensus without additive input noises. Furthermore, Wang and Elia [32] studied how the model parameters affect the appearance of complex behaviour and provided an expression to verify the system stability. Long et al. [33] considered distributed consensus with multiplicative noises and randomly switching graphs assuming that the mean graph is fixed and connected.

In this paper, we consider discrete-time multi-agent distributed averaging algorithms with 
both additive and multiplicative measurement noises under time-varying random graph flows. The time-varying algorithm gain is adopted to attenuate the noises. By constructing difference inequalities of proper stochastic Lyapunov function and tools of algebraic graph theory and martingale convergence theory, we obtain sufficient conditions for the distributed approximation type algorithm to achieve mean square and almost sure average consensus. In detail, we prove that all states of agents converge to a common random variable in mean square and almost surely if the random graph flow is conditionally balanced and uniformly conditionally jointly connected. The mathematical expectation of the variable is right the average of initial states of agents. Moreover, we give an upper bound of the mean square steady-state error quantitatively related to the edge weights, the algorithm gain, the number of agents, the agents' initial states, the second-order moment and the intensity coefficients of the noises. Compared with the relevant literature, main contributions of our paper are summarized as follows.

I. The measurement model covers both cases with additive and multiplicative noises. On one hand, different from the case with only multiplicative noises, due to the introduction of the time-varying algorithm gain to attenuate the additive noises, the closed-loop system becomes a time-varying stochastic system, and the exponential convergence of the expectation of the Lyapunov energy function, which is essential to obtain the almost sure consensus conditions in [13]-[14] and [33], can not be used. On the other hand, different from the case with only additive measurement noises ([11]-[12], [25]), multiplicative noises relying on the relative states between agents make states and noises coupled together in a distributed information structure. This leads to an additional martingale term with coupled states and network graphs in the system centroid equation. The estimation for the term leads to more complex analysis for the closed-loop steady-state error. To these ends, we construct difference inequalities of proper stochastic Lyapunov function. Firstly, by martingale convergence theory, we prove the boundedness of the closed-loop states. Then by substituting the boundedness back into the difference inequality, we obtain mean square average consensus. Furthermore, by tools of martingale convergence theory, we obtain almost sure average consensus. It is worth pointing out that though Wang and Elia ([31]-[32]) considered both additive input noises and Bernoulli fading channels, they used fixed algorithm gain and only obtained mean square weak consensus in absence of the additive input noises. In addition, different from the most existing literature, the noises in this paper are allowed to be spatially and temporally dependent.

II. In this paper, the stochastic Lyapunov flow method is further developed for the case with time-varying random graph flows. Li and Zhang [25] considered deterministic switching 
digraphs and proved that if the network graph flow switches among instantaneously balanced digraphs and is jointly-connected over fixed length consecutive time intervals, then mean square and almost sure consensus are achieved. In Huang [34], the lengths of the time intervals, over which the network is jointly connected, can randomly vary but must be bounded with probability one. In fact, The network graph flow conditions given in [25] and [34] are essentially deterministic type conditions. However, for random graph flows, it is very difficult to verify whether their sample paths satisfy such kinds of conditions with probability one. Particularly, the sample paths of Markovian switching graphs do not satisfy those conditions. In this paper, the network structure among agents is modeled by more general random graph flows. The generalized weighted adjacency matrices are not required to have special statistical properties, such as independency with identical distribution, Markovian switching or stationarity, etc. By introducing the concept of conditional digraph and martingale convergence theory, we establish the uniformly conditionally jointly connected condition to ensure stochastic average consensus. The jointly-connected conditions with respect to i.i.d. graph flows, Markovian and deterministic switching graph flows in the existing literature are all special cases of our condition. Moreover, different from [25], which assumed that the digraphs are balanced, we only require that the conditional digraph is balanced; and different from [27] and [33], we do not require fixed mean graph.

III. In real networks, there exist not only cooperative, but also antagonistic relations between agents ([35]-[37]). Such relations can be modeled by links with positive or negative weights, respectively. In most existing literature on distributed averaging, nonnegative edge weights are required. Liu et al [23] and Touri and Nedic [24] studied noise-free consensus algorithms under random graph flows, and required nonnegative edge weights. Porfiri and Stilwell ([15]) considered noise-free distributed consensus with arbitrary weights in a sampled-data setting, however, the network graph flow is required to be i.i.d. and the mean graph is always connected. In this paper, we show that under the uniformly conditionally jointly connected condition, even though the random edge weights take negative values at some time instants, mean square and almost sure consensus can also be achieved.

The remaining parts of this paper are arranged as follows. Section II gives preliminaries and the problem formulation. Section III gives main results. In Section IV, for two special cases of Markovian switching graph flows with countable states and independently switching graph flows with uncountable states, the sufficient conditions for mean square and almost average consensus are given. Section $\mathrm{V}$ gives the concluding remarks and some future topics.

Notation and symbols: 
$\mathbf{1}_{N}: N$-dimensional vector with all ones;

$\mathbf{0}_{N}, N$-dimensional vector with all zeros;

$I_{N}: N$-dimensional identity matrix;

$O_{m \times n}: m \times n$ dimensional zero matrix;

$\mathbb{R}$ : set of real numbers;

$A \geq B$ : matrix $A-B$ is positive semi-definite;

$A \succeq B$ : matrix $A-B$ is a nonnegative matrix;

$A^{T}$ : transpose of matrix $A$;

$\operatorname{diag}\left(B_{1}, \ldots, B_{n}\right)$ : block diagonal matrix with entries being $B_{1}, \ldots, B_{n}$;

$\|A\|:$ 2-norm of matrix $A$;

$\|A\|_{F}$ : Frobenius-norm of matrix $A$;

$E(\xi)$ : mathematical expectation of random variable $\xi$;

$\operatorname{Var}(\xi)$ : variance of $\xi$;

$|S|$ : the cardinal number of set $S$;

$\lceil x\rceil$ : the minimal integer greater than or equal to real number $x$;

$\lfloor x\rfloor$ : the maximal integer smaller than or equal to $x$;

$b_{n}=O\left(r_{n}\right): \limsup _{n \rightarrow \infty} \frac{\left|b_{n}\right|}{r_{n}}<\infty$, where $\left\{b_{n}, n \geq 0\right\}$ is a real sequence and $\left\{r_{n}, n \geq 0\right\}$ is a positive real sequence;

$b_{n}=o\left(r_{n}\right): \lim _{n \rightarrow \infty} \frac{b_{n}}{r_{n}}=0 ;$

$\mathcal{F}_{\eta}(k)=\sigma(\eta(j), 0 \leq j \leq k), k \geq 0, \mathcal{F}_{\eta}(-1)=\{\Omega, \emptyset\}$, where $\{\eta(k), k \geq 0\}$ is a random vector or matrix sequence.

\section{Preliminaries And Problem Formulation}

\section{A. Preliminaries}

Let the triple $\mathcal{G}=\left\{\mathcal{V}, \mathcal{E}_{\mathcal{G}}, \mathcal{A}_{\mathcal{G}}\right\}$ be a weighted digraph, where $\mathcal{V}=\{1, \ldots, N\}$ is the node set with node $i$ representing agent $i$; $\mathcal{E}_{\mathcal{G}}$ is the edge set, and $(j, i) \in \mathcal{E}_{\mathcal{G}}$ if and only if agent $j$ can send information to agent $i$ directly. Denote the neighbourhood of agent $i$ by $N_{i}=\left\{j \in \mathcal{V} \mid(j, i) \in \mathcal{E}_{\mathcal{G}}\right\}$. We call $\mathcal{A}_{\mathcal{G}}=\left[a_{i j}\right] \in \mathbb{R}^{N \times N}$ the generalized weighted adjacency matrix of $\mathcal{G}$, where $a_{i j} \neq 0 \Leftrightarrow j \in N_{i}$. Since $\mathcal{E}_{\mathcal{G}}$ is uniquely determined by $\mathcal{A}_{\mathcal{G}}$, the digraph can also be denoted by the pair $\mathcal{G}=\left\{\mathcal{V}, \mathcal{A}_{\mathcal{G}}\right\}$. The in-degree and out-degree of agent $i$ are denoted by $\operatorname{deg}_{\text {in }}(i)=\sum_{j=1}^{N} a_{i j}$ and $\operatorname{deg}_{\text {out }}(i)=\sum_{j=1}^{N} a_{j i}$, respectively. We call $L_{\mathcal{G}}=\mathcal{D}_{\mathcal{G}}-\mathcal{A}_{\mathcal{G}}$ the generalized Laplacian matrix of $\mathcal{G}$, where $\mathcal{D}_{\mathcal{G}}=\operatorname{diag}\left(\operatorname{deg}_{i n}(1), \ldots, \operatorname{deg}_{i n}(N)\right)$. By the definition, $L_{\mathcal{G}} \mathbf{1}_{N}=\mathbf{0}_{N}$. If $\operatorname{deg}_{\text {in }}(i)=\operatorname{deg}_{\text {out }}(i), \forall i \in \mathcal{V}$, then $\mathcal{G}$ is balanced. We call $\widetilde{\mathcal{G}}=\left\{\mathcal{V}, \mathcal{E}_{\widetilde{\mathcal{G}}}, \mathcal{A}_{\widetilde{\mathcal{G}}}\right\}$ the reversed digraph of $\mathcal{G}$, where $(i, j) \in \mathcal{E}_{\widetilde{\mathcal{G}}}$ if and only if $(j, i) \in \mathcal{E}_{\mathcal{G}}$ and 
$\mathcal{A}_{\widetilde{\mathcal{G}}}=\mathcal{A}_{\mathcal{G}}^{T}$. Then $\widehat{\mathcal{G}}=\left\{\mathcal{V}, \mathcal{E}_{\mathcal{G}} \cup \mathcal{E}_{\widetilde{\mathcal{G}}}, \frac{\mathcal{A}_{\mathcal{G}}+\mathcal{A}_{\mathcal{G}}^{T}}{2}\right\}$ is called the symmetrized graph of $\mathcal{G}$. Denote $\hat{L}_{\mathcal{G}}=\frac{L_{\mathcal{G}}+L_{\mathcal{G}}^{T}}{2}$. If $a_{i j} \geq 0, \forall i, j \in \mathcal{V}$, then $\mathcal{A}_{\mathcal{G}}$ and $L_{\mathcal{G}}$ defined before degenerate to the weighted adjacency matrix and Laplacian matrix in common sense, and $\hat{L}_{\mathcal{G}}$ is the Laplacian matrix of $\widehat{\mathcal{G}}$ if and only if $\mathcal{G}$ is balanced ([38]).

The union digraph of $\mathcal{G}_{1}=\left\{\mathcal{V}, \mathcal{E}_{\mathcal{G}_{1}}, \mathcal{A}_{\mathcal{G}_{1}}\right\}$ and $\mathcal{G}_{2}=\left\{\mathcal{V}, \mathcal{E}_{\mathcal{G}_{2}}, \mathcal{A}_{\mathcal{G}_{2}}\right\}$ with the common node set $\mathcal{V}$ is denoted by $\mathcal{G}_{1}+\mathcal{G}_{2}=\left\{\mathcal{V}, \mathcal{E}_{\mathcal{G}_{1}} \cup \mathcal{E}_{\mathcal{G}_{2}}, \mathcal{A}_{\mathcal{G}_{1}}+\mathcal{A}_{\mathcal{G}_{2}}\right\}$. By the definition of $L_{\mathcal{G}}$, we know that $L_{\sum_{j=1}^{k} \mathcal{G}_{j}}=\sum_{j=1}^{k} L_{\mathcal{G}_{j}}$. A sequence of edges $\left(i_{1}, i_{2}\right),\left(i_{2}, i_{3}\right), \ldots,\left(i_{k-1}, i_{k}\right)$ is called a directed path from $i_{1}$ to $i_{k}$. If for all $i, j \in \mathcal{V}$, there exists a directed path from $i$ to $j$, then $\mathcal{G}$ is strongly connected.

\section{B. Problem Formulation}

Consider a multi-agent system of $N$ agents whose information structure is described by a time-varying random digraph flow $\left\{\mathcal{G}(k)=\left\{\mathcal{V}, \mathcal{E}_{\mathcal{G}(k)}, \mathcal{A}_{\mathcal{G}(k)}\right\}, k \geq 0\right\}$. We consider the following distributed averaging algorithm:

$$
x_{i}(k+1)=x_{i}(k)+c(k) \sum_{j \in N_{i}(k)} a_{i j}(k)\left(y_{j i}(k)-x_{i}(k)\right), k \geq 0, i \in \mathcal{V},
$$

where $x_{i}(k) \in \mathbb{R}$ is the state of agent $i$ at time instant $k$, and the initial states $x_{i}(0), i=$ $1,2, \ldots, N$ are deterministic variables. Here, $N_{i}(k)$ denotes the neighbourhood of agent $i$ at time instant $k, c(k)$ is the time-varying algorithm gain, and $y_{j i}(k)$ denotes the measurement of agent $j$ 's state by its neighbouring node $i$ at time instant $k$, which is given by

$$
y_{j i}(k)=x_{j}(k)+f_{j i}\left(x_{j}(k)-x_{i}(k)\right) \xi_{j i}(k), i \in \mathcal{V}, j \in N_{i}(k) .
$$

where $\left\{\xi_{j i}(k), k \geq 0\right\}$ represents the measurement noise sequence on channel $(j, i)$ and $f_{j i}\left(x_{j}(k)-x_{i}(k)\right)$ is the noise intensity function. The combination of systems (1) and (2) is called the distributed stochastic approximation type consensus algorithm ([11], [25], [27]). Let $\xi(k)=\left[\xi_{11}(k), \ldots, \xi_{N 1}(k) ; \ldots ; \xi_{1 N}(k), \ldots, \xi_{N N}(k)\right]^{T}$, where $\xi_{j i}(k) \equiv 0$ if $j \notin N_{i}(k)$ all for $k \geq 0$. For the measurement model (2) and the algorithm gain $c(k)$, we have the following assumptions.

(A1) The noise intensity function $f_{j i}(\cdot)$ is a mapping from $\mathbb{R}$ to $\mathbb{R}$. There exist positive constants $\sigma_{j i}$ and $b_{j i}, i, j \in \mathcal{V}$, such that $\left|f_{j i}(x)\right| \leq \sigma_{j i}|x|+b_{j i}, \forall x \in \mathbb{R}$.

(A2) The noise process $\left\{\xi(k), \mathcal{F}_{\xi}(k), k \geq 0\right\}$ is a sequence of vector-valued martingale differences and there exists a positive constant $\beta$ such that $\sup _{k \geq 0} E\left[\|\xi(k)\|^{2} \mid \mathcal{F}_{\xi}(k-1)\right] \leq$ $\beta$ a.s.

(A3) $c(k)>0, \forall k \geq 0, \sum_{k=0}^{\infty} c(k)=\infty, \sum_{k=0}^{\infty} c^{2}(k)<\infty$. 
(A4) $c(k) \downarrow 0, c(k)=O(c(k+h)), k \rightarrow \infty, \forall h>0$.

Remark 1. Assumption (A1) shows that the measurement model (2) covers both cases of additive and multiplicative measurement noises. Here, $b_{j i}, i, j \in \mathcal{V}$ and $\sigma_{j i}, i, j \in \mathcal{V}$ are additive and multiplicative noise intensity coefficients, respectively. This measurement model is suitable for many practical multi-agent systems. For example, in the formation control, to achieve the final desired formation, agents need to get the position information of neighbours. This information acquisition process is usually corrupted by additive noises, such as electromagnetic interference. In addition, the larger the distance between agents is, the more unreliable the received information is, which can be modeled as multiplicative noises in the form of $\sigma_{j i}\left|x_{j}(k)-x_{i}(k)\right| \xi_{j i}(k)$.

The measurement models with additive noises in [11]-[12], [25] and those with multiplicative noises in [13]-[14] and [33] are both special cases of model (2). In detail, the measurement model in [25] is $y_{j i}(k)=x_{j}(k)+\xi_{j i}(k), j \in N_{i}(k)$. The measurement model in [13] is $y_{j i}(k)=$ $x_{j}(k)+f_{j i}\left(x_{j}(k)-x_{i}(k)\right) \xi_{j i}(k), j \in N_{i}(k)$, where $\left|f_{j i}\left(x_{j}(k)-x_{i}(k)\right)\right| \leq \sigma_{j i}\left|x_{j}(k)-x_{i}(k)\right|$. The measurement model in [14] and [33] is $y_{j i}(k)=x_{j}(k)+\sigma_{j i}\left|x_{j}(k)-x_{i}(k)\right| \xi_{j i}(k), j \in N_{i}(k)$. Obviously, the noise intensity functions of the three models above all satisfy (A1).

Remark 2. If only multiplicative measurement noises are considered, existing literature showed that the fixed algorithm gain can ensure strong consensus ([13]-[14]), however, such fixed-gain algorithm can not ensure strong consensus if additive and multiplicative measurement noises co-exist. Here, we adopt the decaying algorithm gain $c(k)$ to attenuate the additive noises. In the field of distributed algorithms, Assumption (A3) ensures that $c(k)$ vanishes with a proper rate for attenuating noises and meanwhile the algorithm does not converge too early.

Let $X(k)=\left[x_{1}(k), \cdots, x_{N}(k)\right]^{T}, D(k)=\operatorname{diag}\left(\alpha_{1}^{T}(k), \ldots, \alpha_{N}^{T}(k)\right)$ with $\alpha_{i}^{T}(k)$ being the $i$ th row of $\mathcal{A}_{\mathcal{G}(k)}, Y(k)=\operatorname{diag}\left(f_{1}(k), \cdots, f_{N}(k)\right)$, where $f_{i}(k)=\operatorname{diag}\left(f_{1 i}\left(x_{1}(k)-x_{i}(k)\right)\right.$, $\left.\cdots, f_{N i}\left(x_{N}(k)-x_{i}(k)\right)\right)$. Substituting (2) into (1) leads to the closed-loop system in the compact form

$$
X(k+1)=\left(I_{N}-c(k) L_{\mathcal{G}(k)}\right) X(k)+c(k) D(k) Y(k) \xi(k) .
$$

Definition 1. ([25]) For systems (1) and (2), iffor any given $X(0) \in \mathbb{R}^{N}$, there exists a random variable $x^{*}$, such that $E\left(x^{*}\right)=\frac{1}{N} \sum_{j=1}^{N} x_{j}(0), \operatorname{Var}\left(x^{*}\right)<\infty, \lim _{k \rightarrow \infty} E\left[x_{i}(k)-x^{*}\right]^{2}=0$, $i \in \mathcal{V}$, and $\lim _{k \rightarrow \infty} x_{i}(k)=x^{*}$ a.s., $i \in \mathcal{V}$, then we say that systems (1) and (2) achieve mean 
square and almost sure average consensus.

In this paper, we aim at giving the conditions under which systems (1) and (2) can achieve mean square and almost sure average consensus based on the models formulated above, i.e., the random digraph flow and the measurement model with both additive and multiplicative noises. The following section gives the main results of this paper.

\section{MAIN RESULTS}

We first introduce the concept of conditional digraphs. We call $E\left[\mathcal{A}_{\mathcal{G}(k)} \mid \mathcal{F}_{\mathcal{A}}(m)\right], m \leq k-1$, the conditional generalized weighted adjacency matrix of $\mathcal{A}_{\mathcal{G}(k)}$ with respect to $\mathcal{F}_{\mathcal{A}}(m)$, and call its associated random graph the conditional digraph of $\mathcal{G}(k)$ with respect to $\mathcal{F}_{\mathcal{A}}(m)$, denoted by $\mathcal{G}(k \mid m)$, i.e., $\mathcal{G}(k \mid m)=\left\{\mathcal{V}, E\left[\mathcal{A}_{\mathcal{G}(k)} \mid \mathcal{F}_{\mathcal{A}}(m)\right]\right\}$.

In this section, we consider the random graph flow with balanced conditional digraphs as follows:

$\Gamma_{1}=\left\{\{\mathcal{G}(k), k \geq 0\} \mid E\left[\mathcal{A}_{\mathcal{G}(k)} \mid \mathcal{F}_{\mathcal{A}}(k-1)\right] \succeq O_{N \times N}\right.$ a.s., $\mathcal{G}(k \mid k-1)$ is balanced a.s., $\left.k \geq 0\right\}$.

We have the following assumption on the random graph flow and the measurement noises.

(A5) The random graph flow $\{\mathcal{G}(k), k \geq 0\}$ and the noise process $\{\xi(k), k \geq 0\}$ are mutually independent.

Let $J_{N}=\frac{1}{N} \mathbf{1 1}^{T}$ and $P_{N}=I_{N}-J_{N}$. Denote the consensus error vector $\delta(k)=P_{N} X(k)$ and the Lyapunov energy function $V(k)=\|\delta(k)\|^{2}$. For any given $k \geq 0$ and positive integer $h$, denote

$$
\lambda_{k}^{h}=\lambda_{2}\left(\sum_{i=k}^{k+h-1} E\left[\hat{L}_{\mathcal{G}(i)} \mid \mathcal{F}_{\mathcal{A}}(k-1)\right]\right),
$$

where $\lambda_{2}(\cdot)$ denotes the second smallest eigenvalue. Since $E\left[\hat{L}_{\mathcal{G}(i)} \mid \mathcal{F}_{\mathcal{A}}(k-1)\right]$ is a real symmetric matrix a.s., $\lambda_{k}^{h}$ is well defined.

We are now in the position for the main result.

Theorem 1. For systems (1)-(2) and the associated random graph flow $\{\mathcal{G}(k), k \geq 0\} \in \Gamma_{1}$, assume that

(a) Assumptions (A1)-(A5) hold;

(b) there exist deterministic positive integer $h$ and positive constants $\theta$ and $\rho_{0}$, such that (b.1) $\inf _{m \geq 0} \lambda_{m h}^{h} \geq \theta$ a.s., (b.2) $\sup _{k \geq 0}\left[E\left[\left\|L_{\mathcal{G}(k)}\right\|^{2^{\max \{h, 2\}}} \mid \mathcal{F}_{\mathcal{A}}(k-1)\right]\right]^{\frac{1}{2^{\max \{h, 2\}}}} \leq \rho_{0}$ a.s.

Then as $k \rightarrow \infty$, the consensus error $\delta(k)$ vanishes in mean square and almost surely, i.e., 
$\lim _{k \rightarrow \infty} E[V(k)]=0$ and $\lim _{k \rightarrow \infty} V(k)=0$, a.s. Moreover, all states $x_{i}(k), i \in \mathcal{V}$, converge to a common random variable $x^{*}$, in mean square and almost surely, with $E\left(x^{*}\right)=\frac{1}{N} \sum_{j=1}^{N} x_{j}(0)$ and

$$
\operatorname{Var}\left(x^{*}\right) \leq \frac{4 c \beta b^{2} \rho_{1}}{N^{2}}+\frac{8 \widetilde{c} \beta \sigma^{2} \rho_{1}}{N^{2}}+\frac{2 c \rho_{2} q_{x}}{N}
$$

where

$$
\begin{aligned}
& c=\sum_{k=0}^{\infty} c^{2}(k), \widetilde{c}=\sum_{k=0}^{\infty} E[V(k)] c^{2}(k), \sigma=\max _{1 \leq i, j \leq N}\left\{\sigma_{j i}\right\}, b=\max _{1 \leq i, j \leq N}\left\{b_{j i}\right\}, \\
& q_{x}=\exp \left\{c \rho_{0}^{2}\right\}\left(\|X(0)\|^{2}+2 c \beta \rho_{1}\left(2 \sigma^{2} q_{v}+b^{2}\right)\right), \\
& q_{v}=\exp \left\{c\left(\rho_{0}^{2}+4 \rho_{1} \beta \sigma^{2}\right)\right\}\left(V(0)+2 c \beta \rho_{1} b^{2}\right), \\
& \rho_{1} \text { and } \rho_{2} \text { are constants satisfying } \\
& \sup _{k \geq 0} E\left[\left|\mathcal{E}_{\mathcal{G}(k)}\right| \max _{1 \leq i, j \leq N} a_{i j}^{2}(k) \mid \mathcal{F}_{\mathcal{A}}(k-1)\right] \leq \rho_{1}, \text { a.s. } \\
& \max _{1 \leq i \leq N} \sup _{k \geq 0} E\left[\left(\sum_{j=1}^{N} a_{i j}(k)-\sum_{j=1}^{N} a_{j i}(k)\right)^{2} \mid \mathcal{F}_{\mathcal{A}}(k-1)\right] \leq \rho_{2}, \text { a.s. }
\end{aligned}
$$

Proof: Firstly, if condition (b.2) holds, noting that

$$
\left|\mathcal{E}_{\mathcal{G}(k)}\right| \max _{1 \leq i, j \leq N} a_{i j}^{2}(k) \leq N(N-1) \max _{1 \leq i, j \leq N} a_{i j}^{2}(k) \leq N(N-1)\|\| L_{\mathcal{G}(k)} \|_{F}^{2},
$$

by the equivalence of 2-norm and Frobenius norm of matrices and the conditional Lyapunov inequality, we know that the deterministic constants $\rho_{1}$ and $\rho_{2}$ are both well defined.

This theorem is proved by 6 Steps as follows.

Step 1: To prove $\sup _{k \geq 0} E[V(k)]<\infty$.

By (3) and the definition of $\delta(k)$, we have

$$
\begin{aligned}
\delta(k+1) & =P_{N}\left(I_{N}-c(k) L_{\mathcal{G}(k)}\right) X(k)+c(k) P_{N} D(k) Y(k) \xi(k) \\
& =\delta(k)-c(k) P_{N} L_{\mathcal{G}(k)} X(k)+c(k) P_{N} D(k) Y(k) \xi(k) .
\end{aligned}
$$

By the definition of $L_{\mathcal{G}(k)}$, it follows that $L_{\mathcal{G}(k)} J_{N}=\mathbf{0}_{N}$, and so $L_{\mathcal{G}(k)} X(k)=L_{\mathcal{G}(k)} \delta(k)$. Then from the above, we have

$$
\delta(k+1)=\left(I_{N}-c(k) P_{N} L_{\mathcal{G}(k)}\right) \delta(k)+c(k) P_{N} D(k) Y(k) \xi(k),
$$

which together with the definition of $V(k)$ leads to

$$
\begin{aligned}
V(k+1) \leq & V(k)-2 c(k) \delta^{T}(k) \frac{L_{\mathcal{G}(k)}^{T} P_{N}^{T}+P_{N} L_{\mathcal{G}(k)}}{2} \delta(k)+c^{2}(k)\left\|L_{\mathcal{G}(k)}\right\|^{2}\|\delta(k)\|^{2} \\
& +c^{2}(k) \xi^{T}(k) Y^{T}(k) D^{T}(k) P_{N} D(k) Y(k) \xi(k) \\
& +2 c(k) \xi^{T}(k) Y^{T}(k) D^{T}(k) P_{N}\left(I_{N}-c(k) P_{N} L_{\mathcal{G}(k)}\right) \delta(k) .
\end{aligned}
$$


By Lemma A.1 and Assumption (A2), we know that

$$
E\left[\xi^{T}(k) Y^{T}(k) D^{T}(k) P_{N}\left(I_{N}-c(k) P_{N} L_{\mathcal{G}(k)}\right) \delta(k)\right]=0 .
$$

Noting that $\mathcal{G}(k \mid k-1)$ is balanced a.s., by Assumption (A5), we get

$$
\begin{aligned}
E\left[\frac{L_{\mathcal{G}(k)}^{T} P_{N}^{T}+P_{N} L_{\mathcal{G}(k)}}{2} \mid \mathcal{F}_{\xi, \mathcal{A}}(k-1)\right] & =E\left[\frac{L_{\mathcal{G}(k)}^{T} P_{N}^{T}+P_{N} L_{\mathcal{G}(k)}}{2} \mid \mathcal{F}_{\mathcal{A}}(k-1)\right] \\
& =E\left[\hat{L}_{\mathcal{G}(k)} \mid \mathcal{F}_{\mathcal{A}}(k-1)\right] \geq O_{N \times N} \text { a.s. }
\end{aligned}
$$

and then by $\delta(k) \in \mathcal{F}_{\xi, \mathcal{A}}(k-1)$, we have

$$
E\left[\delta^{T}(k) \frac{L_{\mathcal{G}(k)}^{T} P_{N}^{T}+P_{N} L_{\mathcal{G}(k)}}{2} \delta(k)\right] \geq 0 .
$$

By Assumption (A1) and the definitions of $Y(k)$ and $V(k)$, we get

$$
\begin{aligned}
\|Y(k)\|^{2} & =\max _{1 \leq i, j \leq N}\left(f_{j i}\left(x_{j}(k)-x_{i}(k)\right)\right)^{2} \\
& \leq \max _{1 \leq i, j \leq N}\left[2 \sigma^{2}\left(x_{j}(k)-x_{i}(k)\right)^{2}+2 b^{2}\right] \\
& \leq 4 \sigma^{2} \max _{1 \leq j, i \leq N}\left[\left(x_{j}(k)-\frac{\sum_{i=1}^{N} x_{i}(k)}{N}\right)^{2}+\left(x_{i}(k)-\frac{\sum_{i=1}^{N} x_{i}(k)}{N}\right)^{2}\right]+2 b^{2} \\
& \leq 4 \sigma^{2} \sum_{j=1}^{N}\left(x_{j}(k)-\frac{\sum_{i=1}^{N} x_{i}(k)}{N}\right)^{2}+2 b^{2} \\
& =4 \sigma^{2} V(k)+2 b^{2} .
\end{aligned}
$$

This together with Assumptions (A2), (A5) and Lemma A.1 gives

$$
\begin{aligned}
& E\left[\xi^{T}(k) Y^{T}(k) D^{T}(k) P_{N} D(k) Y(k) \xi(k)\right] \\
\leq & E\left[\|Y(k)\|^{2}\|\xi(k)\|^{2}\left\|D^{T}(k) D(k)\right\|\right] \\
= & E\left\{E\left[\|Y(k)\|^{2}\|\xi(k)\|^{2}\left\|D^{T}(k) D(k)\right\| \mid \mathcal{F}_{\mathcal{A}, \xi}(k-1)\right]\right\} \\
= & E\left\{\|Y(k)\|^{2} E\left[\|\xi(k)\|^{2} \mid \mathcal{F}_{\mathcal{A}, \xi}(k-1)\right] E\left[\left\|D^{T}(k) D(k)\right\| \mid \mathcal{F}_{\mathcal{A}}(k-1)\right]\right\} \\
\leq & \beta E\left\{\left(4 \sigma^{2} V(k)+2 b^{2}\right) E\left[\left\|D^{T}(k) D(k)\right\| \mid \mathcal{F}_{\mathcal{A}}(k-1)\right]\right\} \\
= & \beta E\left\{\left(4 \sigma^{2} V(k)+2 b^{2}\right) E\left[\lambda_{\max }\left(D^{T}(k) D(k)\right) \mid \mathcal{F}_{\mathcal{A}}(k-1)\right]\right\} \\
= & \beta E\left\{\left(4 \sigma^{2} V(k)+2 b^{2}\right) E\left[\max _{1 \leq i \leq N} \lambda_{\max }\left(\alpha_{i}(k) \alpha_{i}^{T}(k)\right) \mid \mathcal{F}_{\mathcal{A}}(k-1)\right]\right\} \\
= & \beta E\left\{\left(4 \sigma^{2} V(k)+2 b^{2}\right) E\left[\max _{1 \leq i \leq N} \operatorname{tr}\left(\alpha_{i}^{T}(k) \alpha_{i}(k)\right) \mid \mathcal{F}_{\mathcal{A}}(k-1)\right]\right\} \\
\leq & \beta E\left\{\left(4 \sigma^{2} V(k)+2 b^{2}\right) E\left[\left|\mathcal{E}_{\mathcal{G}(k)}\right| \max _{1 \leq i, j \leq N} a_{i j}^{2}(k) \mid \mathcal{F}_{\mathcal{A}}(k-1)\right]\right\} \\
\leq & 4 \sigma^{2} \beta \rho_{1} E[V(k)]+2 b^{2} \beta \rho_{1} .
\end{aligned}
$$

From the above, taking the mathematical expectation on both sides of (7), by (8), (9) and condition (b.2), we get

$$
E[V(k+1)] \leq\left[1+c^{2}(k)\left(\rho_{0}^{2}+4 \beta \sigma^{2} \rho_{1}\right)\right] E[V(k)]+2 b^{2} \beta \rho_{1} c^{2}(k), \quad k \geq 0 .
$$


This together with Assumption (A3) and Lemma A.2 gives that $E[V(k)]$ is bounded (regarding $E[V(k)]$ as $x(k)$ in Lemma A.2).

Step 2: To prove $E[V(k)] \rightarrow 0, k \rightarrow \infty$.

Let $\Phi(m, n)=\left(I_{N}-c(m-1) P_{N} L_{\mathcal{G}(m-1)}\right) \cdots\left(I_{N}-c(n) P_{N} L_{\mathcal{G}(n)}\right), m \geq n, \Phi(n, n)=I_{N}$. From (6) and by iterative calculations, we get

$$
\delta((m+1) h)=\Phi((m+1) h, m h) \delta(m h)+\tilde{\xi}_{m}^{m h}, m \geq 0,
$$

where

$$
\tilde{\xi}_{m}^{m h}=\sum_{j=m h}^{(m+1) h-1} c(j) \Phi((m+1) h, j+1) P_{N} D(j) Y(j) \xi(j) .
$$

By the definition of $V(k)$, it follows that

$$
\begin{aligned}
& V((m+1) h) \\
= & \delta^{T}(m h) \Phi^{T}((m+1) h, m h) \Phi((m+1) h, m h) \delta(m h)+\left(\tilde{\xi}_{m}^{m h}\right)^{T}\left(\tilde{\xi}_{m}^{m h}\right) \\
& +2 \delta^{T}(m h) \Phi^{T}((m+1) h, m h) \tilde{\xi}_{m}^{m h} \\
= & \delta^{T}(m h)\left[\Phi^{T}((m+1) h, m h) \Phi((m+1) h, m h)-I_{N}\right. \\
& \left.+\sum_{i=m h}^{(m+1) h-1} c(i)\left[P_{N} L_{\mathcal{G}(i)}+L_{\mathcal{G}(i)}^{T} P_{N}^{T}\right]\right] \delta(m h) \\
& +V(m h)-\delta^{T}(m h) \sum_{i=m h}^{(m+1) h-1} c(i)\left[P_{N} L_{\mathcal{G}(i)}+L_{\mathcal{G}(i)}^{T} P_{N}^{T}\right] \delta(m h)+\left(\tilde{\xi}_{m}^{m h}\right)^{T}\left(\tilde{\xi}_{m}^{m h}\right) \\
& +2 \delta^{T}(m h) \Phi^{T}((m+1) h, m h) \tilde{\xi}_{m}^{m h} .
\end{aligned}
$$

Noting that $\delta(m h) \in \mathcal{F}_{\xi, \mathcal{A}}(m h-1)$, by the properties of conditional expectation, we know that

$$
\begin{aligned}
& E\left[\delta^{T}(m h) \Phi^{T}((m+1) h, m h) \Phi((m+1) h, j+1) P_{N} D(j) Y(j) \xi(j)\right] \\
= & E\left[\delta ^ { T } ( m h ) E \left[\Phi^{T}((m+1) h, m h) \Phi((m+1) h, j+1)\right.\right. \\
& \left.\left.\times P_{N} D(j) Y(j) \xi(j) \mid \mathcal{F}_{\xi, \mathcal{A}}(j-1)\right]\right], m h \leq j \leq(m+1) h-1, m \geq 0 .
\end{aligned}
$$

By Assumptions (A2), (A5) and Lemma A.1, we have

$$
\begin{aligned}
& E\left[\Phi^{T}((m+1) h, m h) \Phi((m+1) h, j+1) P_{N} D(j) Y(j) \xi(j) \mid \mathcal{F}_{\xi, \mathcal{A}}(j-1)\right] \\
= & E\left[\Phi^{T}((m+1) h, m h) \Phi((m+1) h, j+1) P_{N} D(j) \mid \mathcal{F}_{\xi, \mathcal{A}}(j-1)\right] \\
& \times Y(j) E\left[\xi(j) \mid \mathcal{F}_{\xi, \mathcal{A}}(j-1)\right] \\
= & E\left[\Phi^{T}((m+1) h, m h) \Phi((m+1) h, j+1) P_{N} D(j) \mid \mathcal{F}_{\mathcal{A}}(j-1)\right] \\
& \times Y(j) E\left[\xi(j) \mid \mathcal{F}_{\xi}(j-1)\right] \\
= & \mathbf{0}_{N \times N} .
\end{aligned}
$$


This together with (13) and (15) gives

$$
E\left[\delta^{T}(m h) \Phi^{T}((m+1) h, m h) \tilde{\xi}_{m}^{m h}\right]=0 .
$$

By Assumptions (A3) and (A4), we know that there exist positive integer $m_{0}$ and positive constant $C_{1}$, such that $c^{2}(m h) \leq C_{1} c^{2}((m+1) h), \forall m \geq m_{0}$, and $c(k) \leq 1, \forall k \geq m_{0} h$. By condition (b.2) and the conditional Lyapunov inequality, we obtain that

$$
\sup _{k \geq 0} E\left[\left\|L_{\mathcal{G}(k)}\right\|^{i} \mid \mathcal{F}_{\mathcal{A}}(k-1)\right] \leq \sup _{k \geq 0}\left[E\left[\left\|L_{\mathcal{G}(k)}\right\|^{2^{h}} \mid \mathcal{F}_{\mathcal{A}}(k-1)\right]\right]^{\frac{i}{2^{h}}} \leq \rho_{0}^{i} \text { a.s., } \quad \forall 2 \leq i \leq 2^{h} .
$$

Denote the combinatorial number of choosing $i$ elements from $2 h$ elements by $M_{2 h}^{i}$. By termwise multiplication and using the Hölder inequality repeatedly, noting that

$$
E\left[\left\|L_{\mathcal{G}(k)}\right\|^{l} \mid \mathcal{F}_{\mathcal{A}}(m h-1)\right]=E\left[E\left[\left\|L_{\mathcal{G}(k)}\right\|^{l} \mid \mathcal{F}_{\mathcal{A}}(k-1)\right] \mid \mathcal{F}_{\mathcal{A}}(m h-1)\right], 2 \leq l \leq 2^{h}, k \geq m h
$$

from (17), we have

$$
\begin{aligned}
& E\left\{\left\|\Phi^{T}((m+1) h, m h) \Phi((m+1) h, m h)-I_{N}+\sum_{i=m h}^{(m+1) h-1} c(i)\left(P_{N} L_{\mathcal{G}(i)}+L_{\mathcal{G}(i)}^{T} P_{N}^{T}\right)\right\|\right. \\
& \left.\quad \mid \mathcal{F}_{\mathcal{A}}(m h-1)\right\} \\
\leq & \left(C_{1} \sum_{i=2}^{2 h} M_{2 h}^{i} \rho_{0}^{i}\right) c^{2}((m+1) h) \\
= & C_{1}\left[\left(1+\rho_{0}\right)^{2 h}-1-2 h \rho_{0}\right] c^{2}((m+1) h), m \geq m_{0} .
\end{aligned}
$$

Denote the symmetrized graph of $\mathcal{G}(i \mid m h-1)$ by $\hat{\mathcal{G}}(i \mid m h-1), m h \leq i \leq(m+1) h-1$. Noting that $\mathcal{G}(i \mid i-1)$ is balanced, a.s., we know that $\mathcal{G}(i \mid m h-1)$ is balanced, a.s. Then $E\left[\hat{L}_{\mathcal{G}(i)} \mid \mathcal{F}_{\mathcal{A}}(m h-1)\right]$ is the Laplacian matrix of $\hat{\mathcal{G}}(i \mid m h-1)$, a.s., $m h \leq i \leq(m+1) h-1$. So

$$
\sum_{i=m h}^{(m+1) h-1} E\left[\hat{L}_{\mathcal{G}(i)} \mid \mathcal{F}_{\mathcal{A}}(m h-1)\right] \text { is the Laplacian matrix of } \sum_{i=m h}^{(m+1) h-1} \hat{\mathcal{G}}(i \mid m h-1) \text { a.s. }
$$

Furthermore, by Assumption (A5) and Lemma A.1, we have

$$
\begin{aligned}
& E\left[\delta^{T}(m h)\left[\sum_{i=m h}^{(m+1) h-1} c(i)\left(P_{N} L_{\mathcal{G}(i)}+L_{\mathcal{G}(i)}^{T} P_{N}^{T}\right)\right] \delta(m h)\right] \\
= & 2 E\left[\delta^{T}(m h)\left[\sum_{i=m h}^{(m+1) h-1} c(i) E\left[\hat{L}_{\mathcal{G}(i)} \mid \mathcal{F}_{\xi, \mathcal{A}}(m h-1)\right]\right] \delta(m h)\right] \\
= & 2 E\left[\delta^{T}(m h)\left[\sum_{i=m h}^{(m+1) h-1} c(i) E\left[\hat{L}_{\mathcal{G}(i)} \mid \mathcal{F}_{\mathcal{A}}(m h-1)\right]\right] \delta(m h)\right],
\end{aligned}
$$


which together with Assumption (A4) and condition (b.1) leads to

$$
\begin{aligned}
& E\left[\delta^{T}(m h)\left[\sum_{i=m h}^{(m+1) h-1} c(i)\left(P_{N} L_{\mathcal{G}(i)}+L_{\mathcal{G}(i)}^{T} P_{N}^{T}\right)\right] \delta(m h)\right] \\
\geq & 2 c((m+1) h) E\left[\delta^{T}(m h)\left[\sum_{i=m h}^{(m+1) h-1} E\left[\hat{L}_{\mathcal{G}(i)} \mid \mathcal{F}_{\mathcal{A}}(m h-1)\right]\right] \delta(m h)\right] \\
\geq & 2 c((m+1) h) E\left[\lambda_{m}^{m h} V(m h)\right] \\
\geq & 2 c((m+1) h) E\left[\inf _{m \geq 0}\left(\lambda_{m}^{m h}\right) V(m h)\right] \\
\geq & 2 \theta c((m+1) h) E[V(m h)] \text { a.s. }
\end{aligned}
$$

By Assumptions (A2), (A5) and Lemma A.1, it follows that

$$
\begin{aligned}
& E\left[\xi^{T}(i) Y^{T}(i) D^{T}(i) P_{N} \Phi^{T}((m+1) h, i+1) \Phi((m+1) h, j+1) P_{N} D(j) Y(j) \xi(j)\right] \\
= & E\left[E\left[\xi^{T}(i) Y^{T}(i) D^{T}(i) P_{N} \Phi^{T}((m+1) h, i+1) \Phi((m+1) h, j+1) \mid \mathcal{F}_{\xi, \mathcal{A}}(j)\right]\right. \\
& \left.\times P_{N} D(j) Y(j) \xi(j)\right] \\
= & E\left[E\left[\xi^{T}(i) Y^{T}(i) \mid \mathcal{F}_{\xi, \mathcal{A}}(j)\right]\right. \\
& \left.\times E\left[D^{T}(i) P_{N} \Phi^{T}((m+1) h, i+1) \Phi((m+1) h, j+1) \mid \mathcal{F}_{\mathcal{A}}(j)\right] P_{N} D(j) Y(j) \xi(j)\right] \\
= & E\left[E\left[E\left[\xi^{T}(i) \mid \mathcal{F}_{\xi}(i-1)\right] Y^{T}(i) \mid \mathcal{F}_{\xi, \mathcal{A}}(j)\right]\right. \\
& \left.\times E\left[D^{T}(i) P_{N} \Phi^{T}((m+1) h, i+1) \Phi((m+1) h, j+1) \mid \mathcal{F}_{\mathcal{A}}(j)\right] P_{N} D(j) Y(j) \xi(j)\right] \\
= & 0, i>j
\end{aligned}
$$

which together with the definition of $\tilde{\xi}_{m}^{m h}$ gives

$$
\begin{aligned}
& E\left[\left(\tilde{\xi}_{m}^{m h}\right)^{T}\left(\tilde{\xi}_{m}^{m h}\right)\right]
\end{aligned}
$$

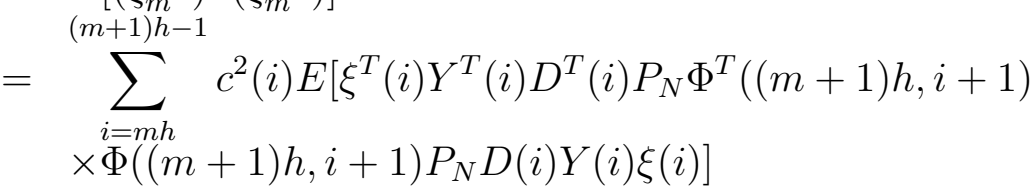

$$
\begin{aligned}
& (m+1) h-1 \\
& \leq \sum_{i=m h} c^{2}(i) E\left\{\left\|\Phi^{T}((m+1) h, i+1) \Phi((m+1) h, i+1)\right\|\left\|D^{T}(i) D(i)\right\|\right. \\
& \left.\times\|Y(i)\|^{2}\|\xi(i)\|^{2}\right\} \\
& =\sum_{i=m h}^{(m+1) h-1} c^{2}(i) E\left\{\| Y ( i ) \| ^ { 2 } E \left[\left\|\Phi^{T}((m+1) h, i+1) \Phi((m+1) h, i+1)\right\|\right.\right. \\
& \left.\left.\times\left\|D^{T}(i) D(i)\right\| \mid \mathcal{F}_{\mathcal{A}}(i-1)\right] E\left[\|\xi(i)\|^{2} \mid \mathcal{F}_{\xi}(i-1)\right]\right\} .
\end{aligned}
$$

By condition (b.2), we know that there is a constant $\rho_{1}^{\prime}$, such that

$$
\sup _{k \geq 0}\left[E\left[\left\|D^{T}(k) D(k)\right\|^{2} \mid \mathcal{F}_{\mathcal{A}}(k-1)\right]\right]^{1 / 2} \leq \rho_{1}^{\prime} \text { a.s. },
$$

which together with the conditional Hölder inequality and Cr-inequality leads to

$$
E\left\{\left\|\Phi^{T}((m+1) h, i+1) \Phi((m+1) h, i+1)\right\|\left\|D^{T}(i) D(i)\right\| \mid \mathcal{F}_{\mathcal{A}}(i-1)\right\}
$$




$$
\begin{aligned}
& \leq \rho_{1}^{\prime}\left\{E\left\{\left\|\Phi^{T}((m+1) h, i+1) \Phi((m+1) h, i+1)\right\|^{2} \mid \mathcal{F}_{\mathcal{A}}(i-1)\right\}\right\}^{\frac{1}{2}} \\
& \leq \rho^{\prime}, m h \leq i \leq(m+1) h-1, m \geq m_{0},
\end{aligned}
$$

where $\rho^{\prime}=\rho_{1}^{\prime}\left\{\left(\sum_{j=0}^{2(h-1)} M_{2(h-1)}^{j}\right) \sum_{l=0}^{2(h-1)} M_{2(h-1)}^{l} \rho_{0}^{2 l}\right\}^{\frac{1}{2}}$. Then by (10), (20) and the above, we get

$$
\begin{aligned}
& \underset{(m+1) h-1}{E\left[\left(\tilde{\xi}_{m}^{m h}\right)^{T}\left(\tilde{\xi}_{m}^{m h}\right)\right]} \\
& \leq \rho^{\prime} \sum_{i=m h} c^{2}(i) E\left\{4 \sigma^{2} V(i) E\left[\|\xi(i)\|^{2} \mid \mathcal{F}_{\xi}(i-1)\right]+2 b^{2} E\left[\|\xi(i)\|^{2} \mid \mathcal{F}_{\xi}(i-1)\right]\right\} \\
& \leq 4 \sigma^{2} \beta \rho^{\prime} \sum_{i=m h}^{(m+1) h-1} c^{2}(i) E[V(i)]+2 b^{2} \beta \rho^{\prime} \sum_{i=m h}^{(m+1) h-1} c^{2}(i), m \geq m_{0} .
\end{aligned}
$$

Finally, by (14), (16), (18), (19) and (21), we have

$$
\begin{aligned}
& E[V((m+1) h)] \\
\leq & \left(1-2 \theta c((m+1) h)+c^{2}((m+1) h) C_{1}\left[\left(1+\rho_{0}\right)^{2 h}-1-2 h \rho_{0}\right]\right) E[V(m h)] \\
& +4 \sigma^{2} \beta \rho^{\prime} \sum_{i=m h}^{(m+1) h-1} c^{2}(i) E[V(i)]+2 b^{2} \beta \rho^{\prime} \sum_{i=m h}^{(m+1) h-1} c^{2}(i), m \geq m_{0} .
\end{aligned}
$$

We call (22) the difference inequality of stochastic Lyapunov function. This together with $\sup _{k \geq 0} E[V(k)]<\infty$ (Step 1) and (22) gives

$$
\begin{aligned}
& E[V((m+1) h)]
\end{aligned}
$$

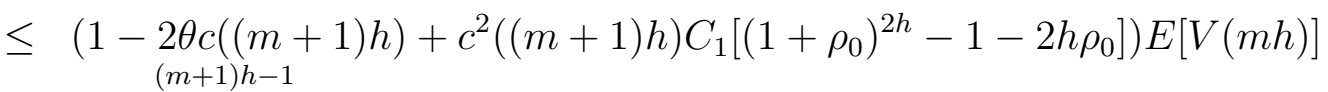

$$
\begin{aligned}
& +C_{2} \sum_{i=m h} c^{2}(i), m \geq m_{0}
\end{aligned}
$$

where $C_{2}=\left(4 \sigma^{2} \sup _{k \geq 0} E[V(k)]+2 b^{2}\right) \beta \rho^{\prime}$.

By Assumption (A3), we know that there exists positive integer $m_{1}$ such that

$$
0<2 \theta c((m+1) h)-c^{2}((m+1) h) C_{1}\left[\left(1+\rho_{0}\right)^{2 h}-1-2 h \rho_{0}\right] \leq 1, \forall m \geq m_{1}
$$

and

$$
\sum_{m=0}^{\infty}\left\{2 \theta c((m+1) h)-c^{2}((m+1) h) C_{1}\left[\left(1+\rho_{0}\right)^{2 h}-1-2 h \rho_{0}\right]\right\}=\infty .
$$

And by Assumption (A4), we get

$$
\lim _{m \rightarrow \infty} \frac{C_{2} \sum_{i=m h}^{(m+1) h-1} c^{2}(i)}{2 \theta c((m+1) h)-c^{2}((m+1) h) C_{1}\left[\left(1+\rho_{0}\right)^{2 h}-1-2 h \rho_{0}\right]}=0 .
$$

Then by Lemma A.3 and (23)-(26), we get $E[V(m h)] \rightarrow 0, m \rightarrow \infty$. Thus, for any given $\epsilon>$ 0 , there exists positive integer $m_{2}$ such that $E[V(m h)]<\epsilon, m \geq m_{2}$, and $\sum_{i=m_{2} h}^{\infty} c^{2}(i)<\epsilon$. 
Let $m_{k}=\left\lfloor\frac{k}{m_{2}}\right\rfloor$. Then for any given $k \geq m_{2} h$, we have $m_{k} \geq m_{2}$ and $0 \leq k-m_{k} h \leq h$. Therefore, by (12), we have

$$
\begin{aligned}
E[V(k+1)] \leq & \prod_{i=m_{k} h}^{k}\left[1+c^{2}(i)\left(\rho_{0}^{2}+4 \rho_{1} \beta \sigma^{2}\right)\right] E\left[V\left(m_{k} h\right)\right] \\
& +2 \rho_{1} b^{2} \beta \sum_{i=m_{k} h}^{k} \prod_{j=i+1}^{k}\left[1+c^{2}(j)\left(\rho_{0}^{2}+4 \rho_{1} \beta \sigma^{2}\right)\right] c^{2}(i) \\
\leq & \exp \left(\left(\rho_{0}^{2}+4 \rho_{1} \beta \sigma^{2}\right) \sum_{i=0}^{\infty} c^{2}(i)\right)\left(1+2 \rho_{1} b^{2} \beta\right) \epsilon, \quad k \geq m_{2} h .
\end{aligned}
$$

where $\prod_{j=k+1}^{k}\left[1+\left(\rho_{0}^{2}+4 \rho_{1} \beta \sigma^{2}\right) c^{2}(j)\right]$ is defined as 1 . Then by the arbitrariness of $\epsilon$, we get

$$
E[V(k)] \rightarrow 0, k \rightarrow \infty
$$

Step 3: To prove $\left\{\frac{1}{N} \sum_{i=1}^{N} x_{i}(k), k \geq 0\right\}$ converges in mean square and almost surely.

Let $\tilde{L}_{\mathcal{G}(k)}=L_{\mathcal{G}(k)}-E\left[L_{\mathcal{G}(k)} \mid \mathcal{F}_{\mathcal{A}}(k-1)\right], k \geq 0$. Noting that the associated digraph of Laplacian matrix $E\left[L_{\mathcal{G}(k)} \mid \mathcal{F}_{\mathcal{A}}(k-1)\right]$ is balanced a.s., we know that $\mathbf{1}^{T} E\left[L_{\mathcal{G}(k)} \mid \mathcal{F}_{\mathcal{A}}(k-1)\right]=$ $\mathbf{0}_{N}^{T}$ a.s. Left multiplied by $\frac{1}{N} \mathbf{1}_{N}^{T}$ on both sides of (3), and then making a summation from 0 to $n-1$ with respect to $k$, we have

$$
\begin{aligned}
\frac{1}{N} \sum_{j=1}^{N} x_{j}(n)= & \frac{1}{N} \sum_{j=1}^{N} x_{j}(0)-\frac{1}{N} \mathbf{1}^{T} \sum_{k=0}^{n-1} c(k) L_{\mathcal{G}(k)} X(k)+\frac{1}{N} \mathbf{1}^{T} \sum_{k=0}^{n-1} c(k) D(k) Y(k) \xi(k) \\
= & \frac{1}{N} \sum_{j=1}^{N} x_{j}(0)-\frac{1}{N} \mathbf{1}^{T} \sum_{k=0}^{n-1} c(k) \tilde{L}_{\mathcal{G}(k)} X(k) \\
& +\frac{1}{N} \mathbf{1}^{T} \sum_{k=0}^{n-1} c(k) D(k) Y(k) \xi(k)
\end{aligned}
$$

Noting that

$$
\begin{aligned}
& E\left[\tilde{L}_{\mathcal{G}(m+i)} X(m+i) \mid \mathcal{F}_{\xi, \mathcal{A}}(m)\right] \\
= & E\left\{E\left[\tilde{L}_{\mathcal{G}(m+i)} X(m+i) \mid \mathcal{F}_{\xi, \mathcal{A}}(m)\right] \mid \mathcal{F}_{\xi, \mathcal{A}}(m+i-1)\right\} \\
= & E\left\{E\left[\tilde{L}_{\mathcal{G}(m+i)} X(m+i) \mid \mathcal{F}_{\xi, \mathcal{A}}(m+i-1)\right] \mid \mathcal{F}_{\xi, \mathcal{A}}(m)\right\} \\
= & E\left\{E\left[\tilde{L}_{\mathcal{G}(m+i)} \mid \mathcal{F}_{\xi, \mathcal{A}}(m+i-1)\right] X(m+i) \mid \mathcal{F}_{\xi, \mathcal{A}}(m)\right\}, 1 \leq i \leq n-m-1,
\end{aligned}
$$

by the definition of $\tilde{L}_{\mathcal{G}(k)}$ and Assumption (A5), it is known that $E\left[\tilde{L}_{\mathcal{G}(k)} \mid \mathcal{F}_{\xi, \mathcal{A}}(k-1)\right]=$ $E\left[\tilde{L}_{\mathcal{G}(k)} \mid \mathcal{F}_{\mathcal{A}}(k-1)\right]=O_{N \times N}, k \geq 0$. Thus, from the above equality, we get

$$
E\left[\tilde{L}_{\mathcal{G}(m+i)} X(m+i) \mid \mathcal{F}_{\xi, \mathcal{A}}(m)\right]=\mathbf{0}_{N}, 1 \leq i \leq n-m-1
$$

which gives

$$
E\left[\sum_{k=0}^{n-1} \tilde{L}_{\mathcal{G}(k)} X(k) \mid \mathcal{F}_{\xi, \mathcal{A}}(m)\right]
$$




$$
\begin{aligned}
& =E\left[\sum_{i=0}^{m} \tilde{L}_{\mathcal{G}(i)} X(i) \mid \mathcal{F}_{\xi, \mathcal{A}}(m)\right]+E\left[\sum_{i=m+1}^{n-1} \tilde{L}_{\mathcal{G}(i)} X(i) \mid \mathcal{F}_{\xi, \mathcal{A}}(m)\right] \\
& =E\left[\sum_{i=0}^{m} \tilde{L}_{\mathcal{G}(i)} X(i) \mid \mathcal{F}_{\xi, \mathcal{A}}(m)\right], \forall m<n-1 .
\end{aligned}
$$

Then by the above and the definition of martingales, we know that $\left\{\frac{1}{N} \mathbf{1}_{N}^{T} \sum_{k=0}^{n} c(k) \tilde{L}_{\mathcal{G}(k)} X(k)\right.$, $\left.\mathcal{F}_{\xi, \mathcal{A}}(n), n \geq 0\right\}$ is a martingale. On the other hand, by (28), we know that

$$
\begin{aligned}
& \sup _{n \geq 0} E\left\|\sum_{k=0}^{n-1} c(k) \tilde{L}_{\mathcal{G}(k)} X(k)\right\|^{2} \\
\leq & \sup _{n \geq 0} \sum_{k=0}^{n-1} c^{2}(k) E\left[\|X(k)\|^{2}\left\|\tilde{L}_{\mathcal{G}(k)}\right\|^{2}\right] \\
\leq & \sup _{k \geq 0} E\left[\left\|\tilde{L}_{\mathcal{G}(k)}\right\|^{2} \mid \mathcal{F}_{\mathcal{A}}(k-1)\right] \sup _{k \geq 0} E\|X(k)\|^{2} \sum_{k=0}^{\infty} c^{2}(k) .
\end{aligned}
$$

By condition (b.2), we know that

$$
\sup _{k \geq 0} E\left[\left\|\tilde{L}_{\mathcal{G}(k)}\right\|^{2} \mid \mathcal{F}_{\mathcal{A}}(k-1)\right]<\infty \text { a.s. }
$$

From (3), (11) and condition (b.2), we get

$$
\begin{aligned}
& E\left[\|X(k+1)\|^{2}\right] \\
= & E\left[X^{T}(k)\left(I_{N}-c(k) L_{\mathcal{G}(k)}^{T}\right)\left(I_{N}-c(k) L_{\mathcal{G}(k)}\right) X(k)\right] \\
& +c^{2}(k) E\left[\xi^{T}(k) Y^{T}(k) D^{T}(k) D(k) Y(k) \xi(k)\right] \\
\leq & E\left[\|X(k)\|^{2}\right]+c^{2}(k) E\left[\|X(k)\|^{2}\left\|L_{\mathcal{G}(k)}\right\|^{2}\right]+c^{2}(k) E\left[\|Y(k)\|^{2}\|\xi(k)\|^{2}\left\|D^{T}(k) D(k)\right\|\right] \\
\leq & E\left[\|X(k)\|^{2}\right]+c^{2}(k) \rho_{0}^{2} E\left[\|X(k)\|^{2}\right]+c^{2}(k) \beta \rho_{1} E\left[4 \sigma^{2} V(k)+2 b^{2}\right] \\
\leq & \left(1+c^{2}(k) \rho_{0}^{2}\right) E\left[\|X(k)\|^{2}\right]+\beta \rho_{1}\left(4 \sigma^{2} \sup _{k \geq 0} E[V(k)]+2 b^{2}\right) c^{2}(k)
\end{aligned}
$$

which together with Lemma A.2 and Assumption (A3) gives $\sup _{k \geq 0} E\left[\|X(k)\|^{2}\right]<\infty$. Then by (29) and (30), we know that

$$
\sup _{n \geq 0} E\left\|\sum_{k=0}^{n-1} c(k) \tilde{L}_{\mathcal{G}(k)} X(k)\right\|^{2}<\infty .
$$

This together with Lemma A.4 leads to

$$
\frac{1}{N} \mathbf{1}_{N}^{T} \sum_{k=0}^{n-1} c(k) \tilde{L}_{\mathcal{G}(k)} X(k) \text { converges a.s. and in } \mathfrak{L}_{2} .
$$

From Assumptions (A2) and (A5), it follows that

$$
E\left[\sum_{k=0}^{n-1} c(k) D(k) Y(k) \xi(k) \mid \mathcal{F}_{\xi, \mathcal{A}}(j)\right]
$$




$$
\begin{aligned}
& =\sum_{k=0}^{j} c(k) D(k) Y(k) \xi(k)+\sum_{k=j+1}^{n-1} E\left[E\left(c(k) D(k) Y(k) \xi(k) \mid \mathcal{F}_{\xi, \mathcal{A}}(k-1)\right) \mid \mathcal{F}_{\xi, \mathcal{A}}(j)\right] \\
& =\sum_{k=0}^{j} c(k) D(k) Y(k) \xi(k), \forall j<n-1 .
\end{aligned}
$$

Thus, the adaptive sequence $\left\{\sum_{j=0}^{n} c(k) D_{\mathcal{G}(k)} Y(k) \xi(k), \mathcal{F}_{\xi, \mathcal{A}}(n), n \geq 0\right\}$ is a martingale. Then by (10) and condition (b.2), we have

$$
\begin{aligned}
& \sup _{n \geq 0} E\left\|\sum_{k=0}^{n-1} c(k) D(k) Y(k) \xi(k)\right\|^{2} \\
= & \sup _{k \geq 0} E\left[\left(\sum_{k=0}^{n-1} c(k) \xi^{T}(k) Y^{T}(k) D^{T}(k)\right)\left(\sum_{k=0}^{n-1} c(k) D(k) Y(k) \xi(k)\right)\right] \\
= & \sup _{k \geq 0} \sum_{k=0}^{n-1} E\left[c^{2}(k) \xi^{T}(k) Y^{T}(k) D^{T}(k) D(k) Y(k) \xi(k)\right] \\
\leq & \beta \sup _{k \geq 0} E\left[\left\|D^{T}(k) D(k)\right\| \mid \mathcal{F}_{\mathcal{A}}(k-1)\right] \sup _{n \geq 0} \sum_{k=0}^{n-1} c^{2}(k) E\|Y(k)\|^{2} \\
\leq & \beta \rho_{1} \sup _{n \geq 0} \sum_{k=0}^{n-1} c^{2}(k)\left(4 \sigma^{2} E[V(k)]+2 b^{2}\right) .
\end{aligned}
$$

By Assumption (A3), the boundedness of $E[V(k)]$ and the above, we get

$$
\sup _{n \geq 0} E\left\|\sum_{k=0}^{n-1} c(k) D(k) Y(k) \xi(k)\right\|^{2}<\infty
$$

which together with Lemma A.4 gives

$$
\frac{1}{N} \mathbf{1}_{N}^{T} \sum_{k=0}^{n-1} c(k) D(k) Y(k) \xi(k) \text { converges, } k \rightarrow \infty \text { a.s. and in } \mathfrak{L}_{2} .
$$

Finally, by (28), (32) and (34) we know that

$$
\frac{1}{N} \sum_{j=1}^{N} x_{j}(n) \longrightarrow x^{*}, n \rightarrow \infty \text { a.s. and in } \mathfrak{L}_{2},
$$

where

$$
x^{*}=\frac{1}{N} \sum_{j=1}^{N} x_{j}(0)-\frac{1}{N} \mathbf{1}_{N}^{T} \sum_{k=0}^{\infty} c(k) \tilde{L}_{\mathcal{G}(k)} X(k)+\frac{1}{N} \mathbf{1}_{N}^{T} \sum_{k=0}^{\infty} c(k) D(k) Y(k) \xi(k) .
$$

Step 4: To prove all $x_{i}(k), i \in \mathcal{V}$ converge to $x^{*}$ as $k \rightarrow \infty$ in mean square and almost surely.

By the definition of $V(k),(27)$ and (35), we have

$$
x_{i}(k) \longrightarrow x^{*}, k \rightarrow \infty, \text { in } \mathfrak{L}_{2}, i \in \mathcal{V} .
$$


Taking conditional expectation on both sides of (7) gives

$$
E\left[V(k+1) \mid \mathcal{F}_{\xi, \mathcal{A}}(k-1)\right] \leq V(k)\left[1+c^{2}(k)\left(\rho_{0}^{2}+4 \sigma^{2} \rho_{1} \beta\right)\right]+2 b^{2} \rho_{1} \beta c^{2}(k) .
$$

Then by Lemma A.2 and Assumption (A3), we obtain

$$
V(k) \rightarrow \text { a finite random variable, } k \rightarrow \infty \text { a.s. }
$$

which together with (27) gives

$$
V(k) \rightarrow 0, k \rightarrow \infty \text { a.s.. }
$$

Then by (35), we have

$$
x_{i}(k) \longrightarrow x^{*}, k \rightarrow \infty \text { a.s., } i \in \mathcal{V} \text {. }
$$

Step 5: To compute the mathematical expectation of $x^{*}$.

By (32), the definition of $\tilde{L}_{\mathcal{G}(k)}$ and Assumption (A5), we have

$$
E\left[\frac{1}{N} \mathbf{1}_{N}^{T} \sum_{k=0}^{\infty} c(k) \tilde{L}_{\mathcal{G}(k)} X(k)\right]=\lim _{n \rightarrow \infty} E\left[\frac{1}{N} \mathbf{1}_{N}^{T} \sum_{k=0}^{n-1} c(k) \tilde{L}_{\mathcal{G}(k)} X(k)\right]=0 .
$$

Similarly, by Assumptions (A2) and (A5), we have

$$
E\left[\frac{1}{N} \mathbf{1}_{N}^{T} \sum_{k=0}^{\infty} c(k) D(k) Y(k) \xi(k)\right]=\lim _{n \rightarrow \infty} E\left[\frac{1}{N} \mathbf{1}_{N}^{T} \sum_{k=0}^{n-1} c(k) D(k) Y(k) \xi(k)\right]=0 .
$$

This together with (36) gives

$$
E\left(x^{*}\right)=\frac{1}{N} \sum_{j=1}^{N} x_{j}(0)
$$

Step 6: To estimate the variance of $x^{*}$.

From (12), by iterative calculations, we have

$$
\begin{aligned}
E[V(k+1)] \leq & \prod_{i=0}^{k}\left[1+\left(\rho_{0}^{2}+4 \beta \sigma^{2} \rho_{1}\right) c^{2}(i)\right] V(0) \\
& +2 \rho_{1} b^{2} \beta \sum_{i=0}^{k} c^{2}(i) \prod_{j=i+1}^{k}\left[1+\left(\rho_{0}^{2}+4 \beta \sigma^{2} \rho_{1}\right) c^{2}(j)\right]
\end{aligned}
$$

where $\prod_{j=k+1}^{k}\left[1+\left(\rho_{0}^{2}+4 \beta \sigma^{2} \rho_{1}\right) c^{2}(j)\right]=1$. Actually, for $\forall k \geq j$,

$$
\prod_{i=j}^{k}\left(1+\left(\rho_{0}^{2}+4 \beta \sigma^{2} \rho_{1}\right) c^{2}(i)\right) \leq \exp \left(\left(\rho_{0}^{2}+4 \beta \sigma^{2} \rho_{1}\right) \sum_{i=j}^{k} c^{2}(i)\right) \leq \exp \left(\left(\rho_{0}^{2}+4 \beta \sigma^{2} \rho_{1}\right) \sum_{i=0}^{\infty} c^{2}(i)\right) \text {. }
$$

This together with (38) leads to

$$
\sup _{k \geq 0} E[V(k)] \leq \exp \left(\left(\rho_{0}^{2}+4 \beta \sigma^{2} \rho_{1}\right) \sum_{i=0}^{\infty} c^{2}(i)\right)\left\{V(0)+2 \rho_{1} b^{2} \beta \sum_{i=0}^{\infty} c^{2}(i)\right\}=q_{v} .
$$


Similarly, by (31) and the above, we have

$$
\begin{aligned}
E\|X(k+1)\|^{2} & \leq\left(1+c^{2}(k) \rho_{0}^{2}\right) E\|X(k)\|^{2}+\beta \rho_{1}\left(4 \sigma^{2} q_{v}+2 b^{2}\right) c^{2}(k) \\
& \leq \exp \left(\rho_{0}^{2} \sum_{k=0}^{\infty} c^{2}(k)\right)\left\{\|X(0)\|^{2}+\beta \rho_{1}\left(4 \sigma^{2} q_{v}+2 b^{2}\right) \sum_{k=0}^{\infty} c^{2}(k)\right\} \\
& =q_{x} .
\end{aligned}
$$

Then by (32), (34), (36), (37), the dominated convergence theorem and Cr-inequality, we have

$$
\begin{aligned}
\operatorname{Var}\left(x^{*}\right)= & E\left[\frac{1}{N} \mathbf{1}_{N}^{T} \sum_{k=0}^{\infty} c(k) D(k) Y(k) \xi(k)-\frac{1}{N} \mathbf{1}_{N}^{T} \sum_{k=0}^{\infty} c(k) \tilde{L}_{\mathcal{G}(k)} X(k)\right]^{2} \\
\leq & 2 E\left[\frac{1}{N} \mathbf{1}_{N}^{T} \sum_{k=0}^{\infty} c(k) D(k) Y(k) \xi(k)\right]^{2}+2 E\left[\frac{1}{N} \mathbf{1}_{N}^{T} \sum_{k=0}^{\infty} c(k) \tilde{L}_{\mathcal{G}(k)} X(k)\right]^{2} \\
\leq & 2 \lim _{n \rightarrow \infty} E\left[\frac{1}{N} \mathbf{1}_{N}^{T} \sum_{k=0}^{n-1} c(k) \tilde{L}_{\mathcal{G}(k)} X(k)\right]^{2} \\
& +2 \lim _{n \rightarrow \infty} E\left[\frac{1}{N} \mathbf{1}_{N}^{T} \sum_{k=0}^{n-1} c(k) D(k) Y(k) \xi(k)\right]^{2}
\end{aligned}
$$

For the first term on right hand side of (41), by the definition of $\tilde{L}_{\mathcal{G}(k)}$, Assumption (A5), Cr-inequality and (40), we have

$$
\begin{aligned}
& \lim _{n \rightarrow \infty} E\left[\frac{1}{N} \mathbf{1}_{N}^{T} \sum_{k=0}^{n-1} c(k) \tilde{L}_{\mathcal{G}(k)} X(k)\right]^{2} \\
= & \lim _{n \rightarrow \infty} E\left[\frac{1}{N} \mathbf{1}_{N}^{T} \sum_{k=0}^{n-1} c(k) L_{\mathcal{G}(k)} X(k)\right]^{2} \\
= & \frac{1}{N^{2}} \lim _{n \rightarrow \infty} \sum_{k=0}^{n-1}\left\{c^{2}(k) E\left[\sum_{i=1}^{N} x_{i}(k)\left(\sum_{j=1}^{N} a_{i j}(k)-\sum_{j=1}^{N} a_{j i}(k)\right)\right]^{2}\right\} \\
\leq & \frac{1}{N} \lim _{n \rightarrow \infty} \sum_{k=0}^{n-1}\left\{c^{2}(k) \sum_{i=1}^{N} E\left[x_{i}^{2}(k)\left(\sum_{j=1}^{N} a_{i j}(k)-\sum_{j=1}^{N} a_{j i}(k)\right)^{2}\right]\right\} \\
\leq & \frac{\rho_{2}}{N} \sum_{k=0}^{\infty} c^{2}(k) E\|X(k)\|^{2} \leq \frac{\rho_{2} q_{x}}{N} \sum_{k=0}^{\infty} c^{2}(k) .
\end{aligned}
$$

For the second term, by Assumption (A2), direct calculations gives

$$
\begin{aligned}
& \lim _{n \rightarrow \infty} E\left[\frac{1}{N} \mathbf{1}_{N}^{T} \sum_{k=0}^{n-1} c(k) D(k) Y(k) \xi(k)\right]^{2} \\
= & \frac{1}{N^{2}} \lim _{n \rightarrow \infty} E\left[\sum_{k=0}^{n-1}\left(\mathbf{1}_{N}^{T} c(k) D(k) Y(k) \xi(k)\right)^{2}\right] \\
\leq & \frac{1}{N^{2}} \lim _{n \rightarrow \infty} \sum_{k=0}^{n-1} c^{2}(k) E\left[\sum_{1 \leq i, j \leq N} \xi_{j i}(k) a_{i j}(k)\left(\sigma_{j i}\left(x_{j}(k)-x_{i}(k)\right)+b_{j i}\right)\right]^{2} .
\end{aligned}
$$


Then by Assumptions (A5), condition (b.2) and Cr-inequality, we have

$$
\begin{aligned}
& \lim _{n \rightarrow \infty} E\left[\frac{1}{N} \mathbf{1}_{N}^{T} \sum_{k=0}^{n-1} c(k) D(k) Y(k) \xi(k)\right]^{2} \\
\leq & \frac{1}{N^{2}} \sum_{k=0}^{\infty}\left\{c^{2}(k) \sum_{(i, j) \in \mathcal{E}_{\mathcal{G}(k)}} E\left[\left|\mathcal{E}_{\mathcal{G}(k)}\right| \xi_{j i}^{2}(k) a_{i j}^{2}(k)\left(\sigma_{j i}\left(x_{j}(k)-x_{i}(k)\right)+b_{j i}\right)^{2}\right]\right\} \\
\leq & \frac{2}{N^{2}} \sum_{k=0}^{\infty}\left\{c^{2}(k) \sum_{(i, j) \in \mathcal{E}_{\mathcal{G}(k)}} E\left[\left|\mathcal{E}_{\mathcal{G}(k)}\right| \xi_{j i}^{2}(k) a_{i j}^{2}(k)\left(\sigma_{j i}^{2}\left(x_{j}(k)-x_{i}(k)\right)^{2}+b_{j i}^{2}\right)\right]\right\} \\
\leq & \frac{2 \beta b^{2} \rho_{1}}{N^{2}} \sum_{k=0}^{\infty} c^{2}(k)+\frac{4 \beta \sigma^{2} \rho_{1}}{N^{2}} \sum_{k=0}^{\infty} E[V(k)] c^{2}(k),
\end{aligned}
$$

which together with (41) and (42) gives (5).

Remark 3. Most of existing literature on consensus-based distributed algorithms assumed that the edge weights, i.e., the entries of $\mathcal{A}_{\mathcal{G}(k)}$, are nonnegative. In this paper, we only assume that the entries of $E\left[\mathcal{A}_{\mathcal{G}(k)} \mid \mathcal{F}_{\mathcal{A}}(k-1)\right]$ are nonnegative almost surely. This relaxation makes the algorithm more flexible at the price of more difficult analysis, since $L_{\mathcal{G}(k)}$ is not a Laplacian matrix any more and some properties of Laplacian matrices are not applicable.

Remark 4. Here, Assumption (A5) requires that the graph flow and the measurement noises are mutually independent. And different from the most existing works on distributed averaging under random network graphs, here, neither the graph flow nor the process of measurement noises is required to be spatially or temporally independent. For the case with time-invariant random graphs, Porfiri and Stilwell [15] and Hatano and Mesbahi [18] assumed independent channels. For the case with time-varying random graphs, Boyd et al. [16], Kar and Moura [17], Tahbaz-Salehi and Jadbabaie [20] and Long et al. [33] assumed that $\{\mathcal{G}(k), k \geq 0\}$ is a sequence of independent random graphs. These spatial or temporal independency requirements can not be always satisfied for real networks. Take a sensor network as the example. On the spatial scale, if a sensor node fails due to battery exhausted, then all channels between this node and its neighbours become inactive. This would happen randomly and the statistics of channels associated with this node are obviously spatially dependent. On the temporal scale, the unreliability of channels would increase due to aging of sensors as time goes on. Thus, the statistics of channels are also temporally dependent. In this paper, we do not require the spatial and temporal independency of the network graphs, which can cover more practical cases. Furthermore, we assume that the overall noises constitute a martingale difference sequence without requiring that the noises are spatial-temporal-independent as in [13]-[14] and [31]- 
[32]).

Remark 5. In [29], the closed-loop system is described by $x(t+1)=A(t) x(t)+B(t) m(t)$, where $\{x(s): s \leq t\}$ is independent of $A(t), B(t)$ and $m(t)$ for all $t \geq 0$; and the disturbance process $m(t)$ is independent of $B(t)$. This assumption obviously fails for our model (3).

Remark 6. We call condition (b.1) $\inf _{m \geq 0} \lambda_{m h}^{h} \geq \theta$ a.s. the uniformly conditionally jointly connected condition, i.e., the conditional digraphs $\mathcal{G}(k \mid k-1)$ are jointly connected over the intervals $[m h,(m+1) h-1], m \geq 0$, and the average algebraic connectivity is uniformly positive bounded away from zero.

Remark 7. The inequality (5) gives a upper bound of the mean square steady-state error. There are three terms on the right hand side of (5), which reflect the impacts of additive noises, multiplicative noises and the instantaneous unbalance of network graph on the final steady-state error, respectively. If the network graph is instantaneously balanced, i.e., $\sum_{j=1}^{N} a_{i j}(k)=\sum_{j=1}^{N} a_{j i}(k), i=1,2, \ldots, N$, a.s., then the third term vanishes. Especially, if the measurement noise sequence $\left\{\xi_{j i}(k), k=0,1, \ldots, i, j=1,2 \ldots, N\right\}$ are both spatially and temporally independent, then from (43), we get

$$
\operatorname{Var}\left(x^{*}\right) \leq \frac{4 c \beta b^{2} \bar{\rho}_{1}}{N^{2}}+\frac{8 \widetilde{c} \beta \sigma^{2} \bar{\rho}_{1}}{N^{2}},
$$

where $\bar{\rho}_{1}$ is a positive constant satisfying $\sup _{k \geq 0} \max _{1 \leq i, j \leq N} E\left[a_{i j}^{2}(k) \mid \mathcal{F}_{\mathcal{A}}(k-1)\right] \leq \bar{\rho}_{1}$, a.s. Moreover, if $\beta=O(N)$ and $\bar{\rho}_{1}=O(1)$ as $N \rightarrow \infty$, then $\operatorname{Var}\left(x^{*}\right)=O(1 / N), N \rightarrow \infty$, which means that the larger the number of sensors is, the higher the accuracy of information fusion is. At the same time, a sensor network with large number of nodes is definitely uneconomic, so there is a trade-off between the performance of the estimation and the cost of the system for selecting the number of nodes.

Remark 8. The constant $\widetilde{c}$ in (5) and (45) can be replaced by $q_{v} c$ from the estimation (39). This removes the term $E[V(k)]$ in $\widetilde{c}$, however, makes the upper bound of the mean square steady-state error more conservative.

\section{SPECIAL CASES}

In this section, we consider two special classes of random graph flows: (i) $\{\mathcal{G}(k), k \geq$ $0\}$ is a Markov chain with countable state space; (ii) $\{\mathcal{G}(k), k \geq 0\}$ is an independent 
process with uncountable state space. By the method of stochastic Lyapunov function based on random graph flows, we obtain sufficient conditions for mean square and almost sure average consensus. For these two special cases, condition (b.1) of Theorem 1 becomes more intuitive and condition (b.2) is weakened.

\section{A. Markovian switching flow}

Definition 2. ([42]) A Markov chain on a countable state space $\mathcal{S}$ with a stationary distribution $\pi$, and transition probability function $\mathbb{P}(x, \cdot)$ is called uniformly ergodic, if there exist positive constants $r>1$ and $R$ such that for all $x \in \mathcal{S}$,

$$
\left\|\mathbb{P}^{n}(x, \cdot)-\pi\right\|_{1} \leq R r^{-n}
$$

Here, $\left\|\mathbb{P}^{n}(x, \cdot)-\pi\right\|_{1}=\sum_{y \in \mathcal{S}}\left|\mathbb{P}^{n}(x, y)-\pi(y)\right|$.

Denote $S_{1}=\left\{A_{j}, j=1,2, \ldots\right\}$, which is a countable set of generalized weighted adjacency matrices and the associated generalized Laplacian matrix of $A_{j}$ by $L_{j}$. Let $\hat{L}_{j}=\frac{L_{j}+L_{j}^{T}}{2}$. In this subsection, we consider the class of random graph flows as below, each element of which is a homogeneous and uniformly ergodic Markov chain with countable states and unique stationary distribution, i.e.

$$
\begin{aligned}
\Gamma_{2}=\{ & \{\mathcal{G}(k), k \geq 0\} \mid\left\{\mathcal{A}_{\mathcal{G}(k)}, k \geq 0\right\} \subseteq S_{1} \text {, and is a homogeneous and uniformly ergodic } \\
& \text { Markov chain with unique stationary distribution } \pi ; E\left[\mathcal{A}_{\mathcal{G}(k)} \mid \mathcal{A}_{\mathcal{G}(k-1)}\right] \succeq O_{N \times N}, \text { a.s., } \\
& \text { and the associated digraph of } \left.E\left[\mathcal{A}_{\mathcal{G}(k)} \mid \mathcal{A}_{\mathcal{G}(k-1)}\right] \text { is balanced a.s., } k \geq 0\right\} .
\end{aligned}
$$

Here, $\pi=\left[\pi_{1}, \pi_{2}, \ldots\right]^{T}, \pi_{j} \geq 0, \sum_{j=1}^{\infty} \pi_{j}=1$, where $\pi_{j}$ denotes $\pi\left(A_{j}\right)$.

We have the following theorem.

Theorem 2. For systems (1)-(2) and the associated random graph flow $\{\mathcal{G}(k), k \geq 0\} \in \Gamma_{2}$, assume that

(i) Assumptions (A1)-(A5) hold;

(ii) the associated graph of the Laplacian matrix $\sum_{j=1}^{\infty} \pi_{j} L_{j}$ contains a spanning tree;

(iii) $\sup _{j \geq 1}\left\|\hat{L}_{j}\right\|<\infty$.

Then systems (1)-(2) achieve mean square and almost sure average consensus.

Proof: Since $\left\{\mathcal{A}_{\mathcal{G}(k)}, k \geq 0\right\}$ is a Markov chain, by the Markov property, we know that $E\left[\mathcal{A}_{\mathcal{G}(k)} \mid \mathcal{F}_{\mathcal{A}}(k-1)\right]=E\left[\mathcal{A}_{\mathcal{G}(k)} \mid \mathcal{A}_{\mathcal{G}(k-1)}\right]$. Thus, $\{\mathcal{G}(k), k \geq 0\} \in \Gamma_{1}$.

By the one-to-one correspondence among $\mathcal{A}_{\mathcal{G}(k)}, L_{\mathcal{G}(k)}$ and $\hat{L}_{\mathcal{G}(k)}$, we know that $\left\{L_{\mathcal{G}(k)}, k \geq\right.$ $0\}$ and $\left\{\hat{L}_{\mathcal{G}(k)}, k \geq 0\right\}$ are both homogeneous and uniformly ergodic Markov chains with the 
unique stationary distribution $\pi$, whose state spaces are $S_{2}=\left\{L_{1}, L_{2}, L_{3}, \ldots\right\}$ and $S_{3}=$ $\left\{\hat{L}_{1}, \hat{L}_{2}, \hat{L}_{3}, \ldots\right\}$, respectively. From (4), we know that

$$
\begin{aligned}
\lambda_{m h}^{h} & =\lambda_{2}\left\{\sum_{i=m h}^{m h+h-1} E\left[\hat{L}_{\mathcal{G}(i)} \mid \hat{L}_{\mathcal{G}(m h-1)}=\hat{L}_{0}\right]\right\} \\
& =\lambda_{2}\left\{\sum_{i=1}^{h} \sum_{j=1}^{\infty} \hat{L}_{j} \mathbb{P}^{i}\left(\hat{L}_{0}, \hat{L}_{j}\right)\right\}, \forall \hat{L}_{0} \in S_{3}, \forall m \geq 0, h \geq 1 .
\end{aligned}
$$

Noting the uniform ergodicity of $\left\{\hat{L}_{\mathcal{G}(k)}, k \geq 0\right\}$ and the uniqueness of the stationary distribution $\pi$, by condition (iii), we have

$$
\begin{aligned}
& \left\|\frac{\sum_{i=1}^{h} \sum_{j=1}^{\infty} \hat{L}_{j} \mathbb{P}^{i}\left(\hat{L}_{0}, \hat{L}_{j}\right)}{h}-\sum_{j=1}^{\infty} \pi_{j} \hat{L}_{j}\right\| \\
= & \left\|\frac{\sum_{i=1}^{h} \sum_{j=1}^{\infty}\left(\hat{L}_{j} \mathbb{P}^{i}\left(\hat{L}_{0}, \hat{L}_{j}\right)-\pi_{j} \hat{L}_{j}\right)}{h}\right\| \\
= & \left\|\frac{\sum_{i=1}^{h} \sum_{j=1}^{\infty} \hat{L}_{j}\left(\mathbb{P}^{i}\left(\hat{L}_{0}, \hat{L}_{j}\right)-\pi_{j}\right)}{h}\right\| \\
\leq & \sup _{j}\left\|\hat{L}_{j}\right\| \frac{\sum_{i=1}^{h} R r^{-i}}{h} \rightarrow 0, h \rightarrow \infty .
\end{aligned}
$$

Furthermore, by the definition of uniform convergence, we know that

$$
\frac{1}{h}\left[\sum_{i=m h}^{m h+h-1} E\left[\hat{L}_{\mathcal{G}(i)} \mid \hat{L}_{\mathcal{G}(m h-1)}\right]\right] \text { converges to } \sum_{j=1}^{\infty} \pi_{j} \hat{L}_{j} \text { a.s. }
$$

uniformly with respect to $m$, as $h \rightarrow \infty$. Denote $\alpha=\lambda_{2}\left(\sum_{j=1}^{\infty} \pi_{j} \hat{L}_{j}\right)$. By condition (ii), it follows that $\alpha>0$. Since the function $\lambda_{2}(\cdot)$, whose arguments are matrices, is continuous, we know that for the given $\frac{\alpha}{2}$, there exists a constant $\delta>0$ such that for any given Laplacian matrix $L,\left|\lambda_{2}(L)-\lambda_{2}\left(\sum_{j=1}^{\infty} \pi_{j} \hat{L}_{j}\right)\right| \leq \frac{\alpha}{2}$, provided $\left\|L-\sum_{j=1}^{\infty} \pi_{j} \hat{L}_{j}\right\| \leq \delta$. Since the convergence is uniform, there exists a positive integer $h_{0}$ such that

$$
\left\|\frac{1}{h}\left[\sum_{i=m h}^{m h+h-1} E\left[\hat{L}_{\mathcal{G}(i)} \mid \hat{L}_{\mathcal{G}(m h-1)}\right]\right]-\sum_{j=1}^{\infty} \pi_{j} \hat{L}_{j}\right\| \leq \delta, h \geq h_{0}, \text { a.s. }
$$

which leads to

$$
\left|\lambda_{2}\left(\frac{1}{h}\left[\sum_{i=m h}^{m h+h-1} E\left[\hat{L}_{\mathcal{G}(i)} \mid \hat{L}_{\mathcal{G}(m h-1)}\right]\right]\right)-\lambda_{2}\left(\sum_{j=1}^{\infty} \pi_{j} \hat{L}_{j}\right)\right| \leq \frac{\alpha}{2}, h \geq h_{0} \text {, a.s. }
$$

Thus,

$$
\lambda_{2}\left(\frac{1}{h}\left[\sum_{i=m h}^{m h+h-1} E\left[\hat{L}_{\mathcal{G}(i)} \mid \hat{L}_{\mathcal{G}(m h-1)}\right]\right]\right) \geq \frac{\alpha}{2}>0, \text { a.s. }
$$


Then by (46), we have $\lambda_{m h}^{h} \geq \frac{h \alpha}{2}>0, h \geq h_{0}$ a.s. So condition (b.1) of Theorem 1 holds. Then by condition (iii), we know that condition (b.2) of Theorem 1 holds. Finally, by Theorem 1 , we get the conclusion of the theorem.

\section{B. Independent graph flow}

Consider the independent graph flow

$$
\begin{array}{r}
\Gamma_{3}=\left\{\{\mathcal{G}(k), k \geq 0\} \mid\{\mathcal{G}(k), k \geq 0\} \text { is an independent process, } E\left[\mathcal{A}_{\mathcal{G}(k)}\right] \succeq O_{N \times N},\right. \text { a.s. } \\
\text { and the associated digraph of } \left.E\left[\mathcal{A}_{\mathcal{G}(k)}\right] \text { is balanced a.s., } k \geq 0\right\} .
\end{array}
$$

We have the following theorem.

Theorem 3. For systems (1)-(2) and the associated random graph flow $\{\mathcal{G}(k), k \geq 0\} \in \Gamma_{3}$, assume that

(i) Assumptions (A1)-(A5) hold;

(ii) there exists a positive integer $h$ such that

$$
\inf _{m \geq 0}\left\{\lambda_{2}\left[\sum_{i=m h}^{(m+1) h-1} E\left[\hat{L}_{\mathcal{G}(i)}\right]\right]\right\}>
$$

(iii) $\sup _{k \geq 0} E\left[\left\|L_{\mathcal{G}(k)}\right\|^{2}\right]<\infty$.

Then systems (1))-(2) achieve mean square and almost sure average consensus.

Proof: From $\mathcal{G}(k) \in \Gamma_{3}$, we know that $\mathcal{G}(k) \in \Gamma_{1}$, and $E\left[\hat{L}_{\mathcal{G}(k)}\right]$ is positive semi-definite. By the independence of $\{\mathcal{G}(k), k \geq 0\}$, we have

$$
E\left[\mathcal{A}_{\mathcal{G}(k)} \mid \mathcal{F}_{\mathcal{A}}(k-1)\right]=E\left[\mathcal{A}_{\mathcal{G}(k)}\right], E\left[L_{\mathcal{G}(k)} \mid \mathcal{F}_{\mathcal{A}}(k-1)\right]=E\left[L_{\mathcal{G}(k)}\right]
$$

which together with Assumption (A5) gives

$$
\begin{aligned}
E\left[\delta^{T}(k) \frac{L_{\mathcal{G}(k)}^{T} P_{N}^{T}+P_{N} L_{\mathcal{G}(k)}}{2} \delta(k)\right] & =E\left[\delta^{T}(k) E\left[\frac{L_{\mathcal{G}(k)}^{T} P_{N}^{T}+P_{N} L_{\mathcal{G}(k)}}{2} \mid \mathcal{F}_{\xi, \mathcal{A}}(k-1)\right] \delta(k)\right] \\
& =E\left[\delta^{T}(k) \frac{E\left[L_{\mathcal{G}(k)}^{T}\right]+E\left[L_{\mathcal{G}(k)}\right]}{2} \delta(k)\right] \\
& =E\left[\delta^{T}(k) E\left[\hat{L}_{\mathcal{G}(k)}\right] \delta(k)\right] \geq 0 .
\end{aligned}
$$

Then similar to the proof of Step 1 of Theorem 1, we get that $E[V(k)]$ is bounded. Denote $\sup _{k \geq 0}\left[E\left[\left\|L_{\mathcal{G}(k)}\right\|^{2}\right]\right]^{\frac{1}{2}}$ by $\rho_{4}$. Since $L_{\mathcal{G}(i)}$ is independent of $L_{\mathcal{G}(j)}, i \neq j$, we do not have to use the conditional Hölder inequality as in (18). Here, by the conditional Lyapunov inequality 
and condition (iii), we have $\sup _{k \geq 0} E\left[\left\|L_{\mathcal{G}(k)}\right\|\right] \leq \sup _{k \geq 0}\left\{E\left[\left\|L_{\mathcal{G}(k)}\right\|^{2}\right]\right\}^{\frac{1}{2}} \leq \rho_{4}$. Then similar to $(18)$, we obtain

$$
\begin{aligned}
& E\left\{\left\|\Phi^{T}((m+1) h, m h) \Phi((m+1) h, m h)-I_{N}+\sum_{i=m h}^{(m+1) h-1} c(i)\left(P_{N} L_{\mathcal{G}(i)}+L_{\mathcal{G}(i)}^{T} P_{N}^{T}\right)\right\|\right\} \\
\leq & \left(C_{1} \sum_{i=2}^{2 h} M_{2 h}^{i} \rho_{4}^{i}\right) c^{2}((m+1) h) \\
= & C_{1}\left[\left(1+\rho_{4}\right)^{2 h}-1-2 h \rho_{4}\right] c^{2}((m+1) h) .
\end{aligned}
$$

Also by the independence of $\{\mathcal{G}(k), k \geq 0\}$ and condition (ii), similarly to (19), we have

$$
\begin{aligned}
& E\left[\delta^{T}(m h) \sum_{i=m h}^{(m+1) h-1} c(i)\left[P_{N} L_{\mathcal{G}(i)}+L_{\mathcal{G}(i)}^{T} P_{N}^{T}\right] \delta(m h)\right] \\
= & 2 E\left[\delta^{T}(m h)\left(\sum_{i=m h}^{(m+1) h-1} c(i) E\left[\hat{L}_{\mathcal{G}(i)}\right]\right) \delta(m h)\right] \\
\geq & 2 c((m+1) h) \inf _{m \geq 0}\left\{\lambda_{2}\left[\sum_{i=m h}^{(m+1) h-1} E\left[\hat{L}_{\mathcal{G}(i)}\right]\right\} E[V(m h)] .\right.
\end{aligned}
$$

Then similarly to the proof of Step 2 of Theorem 1, we get $E[V(k)] \rightarrow 0, k \rightarrow \infty$.

By the independence of $\{\mathcal{G}(k), k \geq 0\}$ and Assumption (A5), we know that the adaptive sequences $\left\{\sum_{j=0}^{n} c(k) D_{\mathcal{G}(k)} Y(k) \xi(k), \mathcal{F}_{\xi, \mathcal{A}}(n), n \geq 0\right\}$ and $\left\{\frac{1}{N} \mathbf{1}^{T} \sum_{k=0}^{n} c(k) \tilde{L}_{\mathcal{G}(k)} X(k)\right.$, $\left.\mathcal{F}_{\xi, \mathcal{A}}(n), n \geq 0\right\}$ are both martingale sequences. Then similar to Steps 3, 4 and 5 of Theorem 1 , we get the conclusion of the theorem.

Remark 9. In Theorem 3, the associated digraph of $E\left[\mathcal{A}_{\mathcal{G}(k)}\right]$, i.e., the mean graph at each time instant, is balanced, so the symmetrized mean graph is undirected. Condition (ii) of Theorem 3 means that the symmetrized mean graphs are jointly-connected (the mean graph has a spanning tree) over consecutive fixed-length time intervals and the average algebraic connectivity is uniformly positive bounded away from zero.

The gossip algorithm ([16]) is a special distributed averaging algorithm with an i.i.d network graph flow. For distributed averaging algorithms with random measurement noises and i.i.d graph flows, the mean square steady-state error can be estimated more precisely with sufficiently small initial algorithm gains. Moreover, the almost sure convergence rate of the $n$-step mean consensus error can be estimated.

Consider the i.i.d graph flow

$$
\Gamma_{4}=\left\{\{\mathcal{G}(k), k \geq 0\} \mid\{\mathcal{G}(k), k \geq 0\} \text { is an i.i.d process, and } E\left[\mathcal{A}_{\mathcal{G}(0)}\right] \succeq O_{N \times N},\right. \text { and }
$$


the associated digraph of $E\left[\mathcal{A}_{\mathcal{G}(0)}\right]$ is balanced $\}$.

Theorem 4. For systems (1)-(2) and the associated random graph flow $\mathcal{G}(k) \in \Gamma_{4}$, assume that

(i) Assumptions (A1)-(A5) hold;

(ii) the associated digraph of the Laplacian matrix $E\left[L_{\mathcal{G}(0)}\right]$ has a spanning tree;

(iii) $E\left[\left\|L_{\mathcal{G}(0)}\right\|^{2}\right]<\infty$.

Then all states $x_{i}(k), i \in \mathcal{V}$, converge to a common random variable $x^{*}$, in mean square and almost surely, with $E\left(x^{*}\right)=\frac{1}{N} \sum_{j=1}^{N} x_{j}(0)$ and

$$
\operatorname{Var}\left(x^{*}\right) \leq \frac{4 c \beta b^{2} \bar{\rho}_{1}}{N^{2}}+\frac{8 \widetilde{c} \beta \sigma^{2} \bar{\rho}_{1}}{N^{2}}+\frac{2 c \bar{\rho}_{2} q_{x}}{N}
$$

where $b, \sigma, c, \widetilde{c}, q_{x}$ are constants defined in (5) and

$$
\bar{\rho}_{1}=E\left[\left|\mathcal{E}_{\mathcal{G}(0)}\right| \max _{1 \leq i, j \leq N} a_{i j}^{2}(0)\right], \bar{\rho}_{2}=\max _{1 \leq i \leq N} E\left[\left(\sum_{j=1}^{N} a_{i j}(0)-\sum_{j=1}^{N} a_{j i}(0)\right)^{2}\right] .
$$

The convergence rate of $n$-step mean consensus error is given by

$$
\frac{1}{n} \sum_{k=0}^{n}\|\delta(k)\|=o\left(\frac{1}{\sqrt{c(n) n}}\right) \text { a.s. }
$$

Furthermore, if the initial algorithm gain is so small that

$$
c(0)<\frac{2 \lambda_{2}\left(E\left[\hat{L}_{\mathcal{G}(0)}\right]\right)}{E\left[\left\|L_{\mathcal{G}(0)}\right\|^{2}\right]+4 \sigma^{2} \beta \bar{\rho}_{1}},
$$

then

$$
\widetilde{c} \leq \frac{c(0) E[V(0)]+2 b^{2} \beta \bar{\rho}_{1} \sum_{k=0}^{\infty} c^{3}(k)}{2 \lambda_{2}\left(E\left[\hat{L}_{\mathcal{G}(0)}\right]\right)-\left(E\left[\left\|L_{\mathcal{G}(0)}\right\|^{2}\right]+4 \sigma^{2} \beta \bar{\rho}_{1}\right) c(0)}
$$

Proof: It is obvious that $\Gamma_{4} \subseteq \Gamma_{3}$, so $\mathcal{G}(k) \in \Gamma_{3}$. By condition (ii) and $\mathcal{G}(k) \in \Gamma_{4}$, we know that $\lambda_{2}\left(E\left[\hat{L}_{\mathcal{G}(0)}\right]\right)>0$ and condition (ii) of Theorem 3 holds with $h=1$. Obviously, condition (iii) together with $\mathcal{G}(k) \in \Gamma_{4}$ implies condition (iii) of Theorem 3 . Then by Theorem 3 , the closed-loop system achieves mean square and almost sure average consensus. From (7), we have

$$
\begin{aligned}
E\left[V(k+1) \mid \mathcal{F}_{\xi, \mathcal{A}}(k)\right] \leq & V(k)-2 c(k) \lambda_{2}\left(E\left[\hat{L}_{\mathcal{G}(0)}\right]\right) V(k)+E\left[\left\|L_{\mathcal{G}(0)}\right\|^{2}\right] c^{2}(k) V(k) \\
& +4 \sigma^{2} \beta \bar{\rho}_{1} c^{2}(k) V(k)+2 b^{2} \beta \bar{\rho}_{1} c^{2}(k) \text { a.s. }
\end{aligned}
$$

which together with $\lambda_{2}\left(E\left[\hat{L}_{\mathcal{G}(0)}\right]\right)>0$ and Lemma A.2 leads to

$$
\sum_{k=0}^{\infty} c(k) V(k)<\infty \text { a.s. }
$$


Then by Assumption (A4) and Kronecker lemma ([39]), we know that

$$
\lim _{n \rightarrow \infty} c(n) \sum_{k=0}^{n} V(k)=0 \text { a.s., }
$$

which together with Cauchy inequality results in (47).

From (50), we have

$$
\begin{aligned}
E[V(k+1)] \leq & E[V(k)]-2 c(k) \lambda_{2}\left(E\left[\hat{L}_{\mathcal{G}(0)}\right]\right) E[V(k)]+E\left[\left\|L_{\mathcal{G}(0)}\right\|^{2}\right] c^{2}(k) E[V(k)] \\
& +4 \sigma^{2} \beta \bar{\rho}_{1} c^{2}(k) E[V(k)]+2 b^{2} \beta \bar{\rho}_{1} c^{2}(k) .
\end{aligned}
$$

Then by Assumption (A4), we have

$$
\begin{aligned}
& \left(2 \lambda_{2}\left(E\left[\hat{L}_{\mathcal{G}(0)}\right]\right)-E\left[\left\|L_{\mathcal{G}(0)}\right\|^{2}\right] c(0)-4 \sigma^{2} \beta \bar{\rho}_{1} c(0)\right) c^{2}(k) E[V(k)] \\
\leq & c(k) E[V(k)]-c(k+1) E[V(k+1)]+2 b^{2} \beta \bar{\rho}_{1} c^{3}(k) .
\end{aligned}
$$

Take summation on both sides of the above inequality from $k=0$ to $k=n$ gives

$$
\begin{aligned}
& \left(2 \lambda_{2}\left(E\left[\hat{L}_{\mathcal{G}(0)}\right]\right)-E\left[\left\|L_{\mathcal{G}(0)}\right\|^{2}\right] c(0)-4 \sigma^{2} \beta \bar{\rho}_{1} c(0)\right) \sum_{k=0}^{n} c^{2}(k) E[V(k)] \\
\leq & c(0) E[V(0)]-c(n+1) E[V(n+1)]+2 b^{2} \beta \bar{\rho}_{1} \sum_{k=0}^{n} c^{3}(k) .
\end{aligned}
$$

Then by (48) and let $n \rightarrow \infty$, we have (49).

\section{CONCLUSION}

We have considered discrete-time stochastic approximation type distributed averaging algorithms with random measurement noises and time-varying random graph flows. Compared with the existing literature, our model is more widely applicable in the sense that i) the measurement covers both additive and multiplicative noises; ii) the network graphs and noises are not required to be spatially and temporally independent; iii) the edge weights of network graphs are not necessarily nonnegative with probability one. By constructing difference inequalities of proper stochastic Lyapunov function, the algebraic graph theory and martingale convergence theory, sufficient conditions have been given to achieve mean square and almost sure average consensus. It has been shown that all states of agents converge to a common variable in mean square and almost surely if the network graph flow is conditionally balanced and uniformly conditionally jointly connected. The mathematical expectation of the common variable is right the average of initial values. Moreover, an upper bound of the mean square steady-state error has been given in relation to the edge weights, the time-varying algorithm gain, the number of agents, the agents' initial values, the second-order moment and the intensity coefficients of noises. Especially, if the measurement noises are both spatially 
and temporally independent, then the mean square steady-state error vanishes as the number of nodes increases to infinity under mild conditions on the network graphs.

Convergence rate is an important performance for distributed averaging algorithms. Different from the fixed-gain algorithms for noise-free cases ([19], [30], [44]), here, the non-zero off-diagonal elements of the closed-loop state matrix are not uniformly bounded away from zero, which results in much more difficulties to get the exact stochastic convergence rates of the algorithm. For the case with i.i.d graph flows, we have given a rough estimate for the $n$-step mean consensus error with probability one. It is interesting to develop effective tools to give the exact stochastic convergence rates of our algorithms.

\section{APPENDIX}

Lemma A.1. Let $\left\{Z_{k}, k \geq 0\right\}$ and $\left\{W_{k}, k \geq 0\right\}$ be mutually independent random vector sequences. Then $\sigma\left(Z_{j}, Z_{j+1}, \ldots\right)$ and $\sigma\left(W_{j}, W_{j+1}, \ldots\right)$ are conditionally independent given $\sigma\left(Z_{0}, \ldots, Z_{j-1}, W_{0}, \ldots, W_{j-1}\right), \forall j \geq 1$.

Proof: Denote $Z_{m \sim n}=\left\{Z_{m}=z_{m}, \ldots, Z_{n}=z_{n}\right\}$ and $Z_{m \sim \infty}=\left\{Z_{m}=z_{m}, Z_{m+1}=z_{m+1}, \ldots\right\}$ where $z_{k}$ denotes the possible values of $Z_{k}$. By the definition of conditional probability,

$$
\begin{aligned}
& \mathbb{P}\left\{Z_{j \sim \infty}, W_{j \sim \infty} \mid Z_{0 \sim j-1}, W_{0 \sim j-1}\right\} \\
= & \mathbb{P}\left\{W_{j \sim \infty} \mid Z_{0 \sim j-1}, W_{0 \sim j-1}\right\} \mathbb{P}\left\{Z_{j \sim \infty} \mid Z_{0 \sim j-1}, W_{0 \sim \infty}\right\} .
\end{aligned}
$$

Noting that $\sigma\left(Z_{0 \sim \infty}\right)=\sigma\left(\sigma\left(Z_{j \sim \infty}\right) \cup \sigma\left(Z_{0 \sim j-1}\right)\right)$ and $\sigma\left(Z_{0 \sim \infty}\right)$ is independent of $\sigma\left(W_{0 \sim \infty}\right)$, by Corollary 3 of Section 7.3 of [39], we have

$$
\mathbb{P}\left\{Z_{j \sim \infty} \mid Z_{0 \sim j-1}, W_{0 \sim \infty}\right\}=\mathbb{P}\left\{Z_{j \sim \infty} \mid Z_{0 \sim j-1}\right\}=\mathbb{P}\left\{Z_{j \sim \infty} \mid Z_{0 \sim j-1}, W_{0 \sim j-1}\right\},
$$

which together with (A.1) gives

$\mathbb{P}\left\{Z_{j \sim \infty}, W_{j \sim \infty} \mid Z_{0 \sim j-1}, W_{0 \sim j-1}\right\}=\mathbb{P}\left\{W_{j \sim \infty} \mid Z_{0 \sim j-1}, W_{0 \sim j-1}\right\} \mathbb{P}\left\{Z_{j \sim \infty} \mid Z_{0 \sim j-1}, W_{0 \sim j-1}\right\}$.

By the definition of conditional independence, we get the conclusion.

Lemma A.2. ([43]) Let $\{x(k), \mathcal{F}(k)\},\{\alpha(k), \mathcal{F}(k)\},\{\beta(k), \mathcal{F}(k)\}$ and $\{\gamma(k), \mathcal{F}(k)\}$ be nonnegative adaptive sequences satisfying

$$
E(x(k+1) \mid \mathcal{F}(k)) \leq(1+\alpha(k)) x(k)-\beta(k)+\gamma(k), k \geq 0 \text { a.s. }
$$

and $\sum_{k=0}^{\infty}(\alpha(k)+\gamma(k))<\infty$ a.s. then $x(k)$ converges to a finite random variable a.s., and $\sum_{k=0}^{\infty} \beta(k)<\infty$ a.s. 
Lemma A.3. ([40]) Let $\{u(k), k \geq 0\},\{q(k), k \geq 0\}$ and $\{\alpha(k), k \geq 0\}$ be real sequences, where $0<q(k) \leq 1, \alpha(k) \geq 0, k \geq 0, \sum_{k=0}^{\infty} q(k)=\infty, \frac{\alpha(k)}{q(k)} \rightarrow 0, k \rightarrow \infty$, and $u(k+1) \leq$ $(1-q(k)) u(k)+\alpha(k)$. Then $\limsup _{k \rightarrow \infty} u(k) \leq 0$. Especially, if $u(k) \geq 0, k \geq 0$, then $u(k) \rightarrow 0, k \rightarrow \infty$.

Lemma A.4. ([41]) Let $\{X(k), \mathcal{F}(k)\}$ be a martingale sequence satisfying $\sup _{k \geq 0} E\left[\|X(k)\|^{2}\right]<$ $\infty$. Then $X(k)$ converges in mean square and almost surely.

\section{REFERENCES}

[1] N. Lynch, Distributed Algorithms. San Matero, CA: Morgan Kaufmann, 1996.

[2] L. Xiao and S. Boyd, "Fast linear iterations for distributed averaging," Systems and Control Letters, vol. 53, no. 1, pp. 65-78, 2004.

[3] R. Olfati-Saber and P. Jalalkamali, "Coupled distributed estimation and control for mobile sensor networks," IEEE Transactions on Automatic Control, vol. 57, no. 10, pp. 2609-2614, 2012.

[4] A. T. Kamal, J. A. Farrell and A. K. Roy-Chowdhury, "Information weighted consensus filters and their application in distributed camera networks," IEEE Transactions on Automatic Control,vol. 58, no. 12, pp. 3112-3125, 2013.

[5] L. Xiao, S. Boyd and S. Lall, "A scheme for robust distributed sensor fusion based on average consensus," Proc. 4th International Symposium on Information Processing in Sensor Networks, April 15, pp. 63-70, 2005.

[6] A. H. Sayed, "Adaptive Networks," Proceedings of IEEE, vol. 102, no. 4, pp. 460-497, 2014.

[7] J. Tsitsiklis, D. Bertsekas and M. Athans, "Distributed asynchronous deterministic and stochastic gradient optimization algorithms," IEEE Transactions on Automatic Control, vol. 31, no. 9, pp. 803-812, 1986.

[8] N. Amelina, A. Fradkov, Y. Jiang and D. J. Vergados, "Approximate consensus in stochastic networks with application to load balancing," IEEE Transactions on Information Theory, vol. 61, no. 4, pp. 1739-1752, 2015.

[9] N. Elia, "Remote stabilization over fading channels," Systems and Control Letters, vol. 54, no. 3, pp. 237-249, 2005.

[10] V. S. Frost, J. A. Stiles, K. S. Shanmugan and J. C. Holtzman, "A model for radar images and its application to adaptive digital filtering of multiplicative noise," IEEE Transactions on Pattern Analysis and Machine Intelligence, vol. PAMI-4, no. 2, pp. 157-166, 1982.

[11] M. Huang and J. H. Manton, "Coordination and consensus of networked agents with noisy measurements: stochastic algorithms and asymptotic behavior," SIAM Journal on Control and Optimization, vol. 48, no. 1, pp. 134-161, 2009.

[12] T. Li and J. F. Zhang, "Mean square average-consensus under measurement noises and fixed topologies: Necessary and sufficient conditions," Automatica, vol. 45, no. 8, pp. 1929-1936, 2009.

[13] T. Li, F. Wu and J. F. Zhang, "Multi-agent consensus with relative-state-dependent measurement noises," IEEE Transactions on Automatic Control, vol. 59, no. 9, pp. 2463-2468, 2014. 
[14] Y. H. Ni and X. Li, "Consensus seeking in multi-agent systems with multiplicative measurement noises," Systems and Control Letters, vol. 62, no. 5, pp. 430-437, 2013.

[15] M. Porfiri and D. J. Stilwell, "Consensus seeking over random weighted directed graphs," IEEE Transactions on Automatic Control, vol. 52, no. 9, pp. 1767-1773, 2007.

[16] S. Boyd, A. Ghosh, B. Prabhakar and D. Shah,"Randomized gossip algorithms," IEEE Transactions on Information Theory, vol. 52, no. 6, pp. 2508-2530, 2006.

[17] S. Kar and J. M. F. Moura, "Sensor networks with random links: Topology design for distributed consensus," IEEE Transactions on Signal Processing, vol. 56, no. 7, pp. 3315-3326, 2008.

[18] Y. Hatano and M. Mesbahi, "Agreement over random networks," IEEE Transactions on Automatic Control, vol. 50, no. 11, pp. 1867-1872, 2005.

[19] D. Bajović, J. Xavier, J. M. F. Moura and B. Sinopoli, "Consensus and products of random stochastic matrices: Exact rate for convergence in probability," IEEE Transactions on Signal Processing, vol. 61, no. 10, pp. 2557-2571, 2013.

[20] A. Tahbaz-Salehi and A. Jadbabaie, "A necessary and sufficient condition for consensus over random networks," IEEE Transactions on Automatic Control, vol. 53, no. 3, pp.791-795, 2008.

[21] A. Tahbaz-Salehi and A. Jadbabaie, "Consensus over ergodic stationary graph processes," IEEE Transactions on Automatic Control, vol. 55, no. 1, pp. 225-230, 2010.

[22] I. Matei, J. S. Baras and C. Somarakis, "Convergence results for the linear consensus problem under Markovian random graphs," SIAM Journal on Control and Optimization, vol. 51, no. 2, 1574-1591, 2013.

[23] B. Liu, W. Lu and T. Chen, "Consensus in networks of multiagents with switching topologies modeled as adapted stochastic processes," SIAM Journal on Control and Optimization, vol. 49, no. 1, pp. 227-253, 2011.

[24] B. Touri and A. Nedic, "Product of random stochastic matrices," IEEE Transactions on Automatic Control, vol. 59, no. 2, pp. 437-448, 2014.

[25] T. Li and J. F. Zhang, "Consensus conditions of multi-agent systems with time-varying topologies and stochastic communication noises," IEEE Transactions on Automatic Control, vol. 55, no. 9, pp. 2043-2057, 2010.

[26] R. Rajagopal and M. J. Wainwright, "Networked-based consensus averaging with general noisy channels," IEEE Transactions on Signal Processing, vol. 59, no. 1, pp. 373-385, 2011.

[27] S. Kar and J. M. F. Moura, "Distributed consensus algorithms in sensor networks with imperfect communication: link failures and channel noise.” IEEE Transactions on Signal Processing, vol. 57, no. 1, pp. 355-369, 2009.

[28] M. Huang, S. Dey, G. N. Nair and J. H. Manton, "Stochastic consensus over noisy networks with Markovian and arbitrary switches," Automatica, vol. 46, no. 10, pp. 1571-1583, 2010.

[29] T. C. Aysal and K. E. Barner, "Convergence of consensus models with stochastic distrubances," IEEE Transactions on Information Theory, vol. 56, no. 8, pp. 4101-4113, 2010.

[30] S. Patterson, B. Bamieh and A. E. Abbadi, "Convergence rates of distributed average consensus with stochastic link failures," IEEE Transactions on Automatic Control, vol. 55, no. 4, pp. 880-892, 2010.

[31] J. Wang and N. Elia, "Distributed averaging under constraints on information exchange: emergence of levy 
flights," IEEE Transactions on Automatic Control, vol. 57, no. 10, pp. 2435-2449, 2012.

[32] J. Wang and Nicola Elia, "Mitigation of complex behavior over networked systems: analysis of spatially invariant structures," Automatica, vol. 49, no. 6, pp. 1626-1638, 2013.

[33] Y. Long, S. Liu and L. Xie, "Distributed consensus of discrete-time multi-agent systems with multiplicative noises," International Journal of Robust and Nonlinear Control, vol. 38, no. 2, pp. 3113-3131, 2014.

[34] M. Huang, "Stochastic approximation for consensus: A new approach via ergodic backward products," IEEE Transactions on Automatic Control, vol. 57, no. 12, pp. 2994-3008, 2012.

[35] D. Easley and J. Kleinberg, Networks, Crowds and Markets. Reasoning About a Highly Connected World. Cambridge, U.K.: Cambridge University Press, 2010.

[36] S. Wasserman and K. Faust, Social Network Analysis: Methods and Applications. Cambridge, U.K.: Cambridge University Press, 1994.

[37] C. Altafini, "Consensus problems on networks with antagonistic interactions," IEEE Transactions on Automatic Control, vol. 58, no. 4, pp. 935-946, 2013.

[38] R. Olfati-Saber and R. M. Murray, "Consensus problem in networks of agents with switching topology and time-delays," IEEE Trans. on Automatic Control, Vol. 49, No. 9, pp. 1520-1533, 2004.

[39] Y. S. Chow and H. Teicher, Probability Theory: Independence, Interchangeability, Martingales. 3rd Edition. New York: Springer-Verlag, 1997.

[40] B. T. Polyak, Introduction to Optimization. New York: Optimization Software Inc., 1987.

[41] R. B. Ash, Real Analysis and Probability. New York: Academic Press, 1972.

[42] S. P. Meyn and R. L. Tweedie, Markov Chains and Stochastic Stability. London: Springer-Verlag, 1993.

[43] H. Robbins and D. Siegmund, A convergence theorem for non negative almost supermartingales and some applications. In Herbert Robbins Selected Papers Ed. T. L. Lai, and D. Siegmund, New York: SpringerVerlag, 1985.

[44] A. Olshevsky and J. N. Tsitisklis, "Convergence speed in distributed consensus and averaging," SIAM Journal of Control and Optimization, vol. 48, no. 1, pp. 33-55, 2009. 